Proceedings of the Edinburgh Mathematical Society (2004) 47, 597-624 (C)

DOI:10.1017/S0013091503000142 Printed in the United Kingdom

\title{
PROBABILISTIC INTERPRETATION AND PARTICLE METHOD FOR VORTEX EQUATIONS WITH NEUMANN'S BOUNDARY CONDITION
}

\author{
BENJAMIN JOURDAIN ${ }^{1}$ AND SYLVIE MÉLÉARD ${ }^{2}$ \\ ${ }^{1}$ ENPC-CERMICS, 6-8 av. Blaise Pascal, Cité Descartes, Champs sur Marne, \\ 77455 Marne-la-Vallée Cedex 2, France (jourdain@cermics.enpc.fr) \\ ${ }^{2}$ Université Paris 10, MODALX, 200 av. de la République, 92000 Nanterre
}

(sylvie.meleard@u-paris10.fr)

(Received 15 February 2003)

\begin{abstract}
We are interested in proving the convergence of Monte Carlo approximations for vortex equations in bounded domains of $\mathbb{R}^{2}$ with Neumann's condition on the boundary. This work is the first step towards justifying theoretically some numerical algorithms for Navier-Stokes equations in bounded domains with no-slip conditions.

We prove that the vortex equation has a unique solution in an appropriate energy space and can be interpreted from a probabilistic point of view through a nonlinear reflected process with space-time random births on the boundary of the domain.

Next, we approximate the solution $w$ of this vortex equation by the weighted empirical measure of interacting diffusive particles with normal reflecting boundary conditions and space-time random births on the boundary. The weights are related to the initial data and to the Neumann condition. We prove a trajectorial propagation-of-chaos result for these systems of interacting particles. We can deduce a simple stochastic particle algorithm to simulate $w$.
\end{abstract}

Keywords: vortex equation on a bounded domain; Monte Carlo approximation; interacting particle systems with reflection; space-time random births

2000 Mathematics subject classification: Primary 60K35; 76D05

\section{Introduction}

We are interested in proving the convergence of Monte Carlo approximations for vortex equations in bounded domains of $\mathbb{R}^{2}$ with Neumann's condition on the boundary. This work is the first step towards justifying theoretically some numerical algorithms for Navier-Stokes equations in bounded domains with no-slip conditions, as proposed by Chorin or Cottet. To our knowledge, there was no proof of convergence of such particle methods, even for the deterministic ones, and even in the simplified case we consider in this paper.

We consider the Navier-Stokes equation which describes the evolution of the velocity field of an incompressible viscous fluid in a bounded domain $\Theta$ of $\mathbb{R}^{2}$ satisfying the no-slip 
boundary condition:

$$
\begin{gathered}
\partial_{t} u(t, x)+(u \cdot \nabla) u(t, x)=\nu \Delta u(t, x)-\nabla p \quad \text { in } \Theta, \\
\nabla \cdot u(t, x)=0 \quad \text { in } \Theta, \quad u(t, x)=(0,0) \quad \text { for } x \in \partial \Theta,
\end{gathered}
$$

where $p$ is the pressure and $\nu>0$ the viscosity coefficient. Our aim is to obtain a probabilistic interpretation of this equation which enables us to construct an efficient Monte Carlo particle method for the simulation of the solutions. Another probabilistic approach based on branching processes has already been developed by Benachour, Roynette and Vallois [2], generalized in dimension 3 by Giet [10]. But even if the authors propose some particle approximations, the convergence of the method is not shown and the particle systems they describe are not of practical use. Our purpose is to construct some easily simulable particle systems, behaving as diffusion processes reflected on the boundary, with space-time random births located at the boundary and to prove rigorously the propagation of chaos of the laws of these processes to a probability measure associated with the solution of the Navier-Stokes equation.

One associates classically with the two-dimensional Navier-Stokes equation in the whole plane the simpler vortex equation satisfied by the curl of the velocity. This equation behaves as a McKean-Vlasov equation and justifies the famous vortex simulation algorithm due to Chorin [6], the velocity being obtained as the convolution of the vorticity by the Biot-Savart kernel. The probabilistic approach, first introduced by Marchioro and Pulvirenti [17], has been developed in Méléard $[\mathbf{1 9}, \mathbf{2 0}]$.

In a bounded domain, a similar approach would consist of replacing the Biot-Savart kernel by the orthogonal gradient $K$ of the Green function for the Dirichlet problem in the domain. By this approach, one only obtains the nullity of the normal component of the velocity of the fluid at the boundary. As pointed out by Chorin in his algorithm [6], vorticity has to be produced at the boundary to ensure the nullity of the tangential component. Several authors $[\mathbf{1}, \mathbf{3}, \mathbf{7}, \mathbf{8}, \mathbf{2 6}]$ have given different mathematical frameworks to describe this creation of vorticity. The non-local Neumann boundary condition proposed by Cottet [7] seems the most suitable formulation for a probabilistic interpretation. But, unhappily, to our knowledge, no energy estimate is available for this formulation, which makes its analysis difficult.

As a first step, in this paper, we deal with a fixed Neumann condition and consider the vortex equation

$$
\begin{gathered}
\partial_{t} w(t, x)+\nabla \cdot(w K w)(t, x)=\nu \Delta w(t, x) \quad \text { in } \Theta, \\
w(0, x)=w_{0}(x) \quad \text { in } \Theta, \\
\partial_{n} w=\nabla w \cdot n=g \quad \text { on } \partial \Theta,
\end{gathered}
$$

where the function $g$ is fixed. We will in particular see that this Neumann condition is represented in our probabilistic framework by space-time random births located at the boundary of the domain.

More generally, in this paper, we prove the existence of a unique solution to this vortex equation with Neumann's boundary condition, in an appropriate space for which we 
have obtained energy a priori estimates. Then we associate with the solution a nonlinear diffusive and reflected process, with space-time random birth (with a law depending on the functions $g$ and $w_{0}$ ). We construct interacting normally reflected particle systems with space-time random births and prove the propagation of chaos following the law of the nonlinear process previously defined. We are inspired by the paper of Sznitman [23], which concerns the behaviour of interacting and reflected McKean-Vlasov particle systems living in a bounded domain. Some additional difficulties appear here, due to the singular interacting kernel $K$ and to the space-time random births. Moreover, the interaction is mean-field but is a function of the weighted empirical measure, the weights being related to the initial condition and to $g$.

\section{The model}

Let $T>0$. We are interested in the following equation:

$$
\left.\begin{array}{c}
\left.\left.\partial_{t} w(t, x)+\nabla \cdot(w K w)(t, x)=\nu \Delta w(t, x) \quad \text { in }\right] 0, T\right] \times \Theta, \\
w(0, x)=w_{0}(x) \quad \text { in } \Theta, \\
\left.\left.\partial_{n} w=\nabla w \cdot n=g \quad \text { on }\right] 0, T\right] \times \partial \Theta,
\end{array}\right\}
$$

where $n(x)$ denotes the outward normal to $\partial \Theta$ at the point $x$ and

$$
K w(t, x)=\int_{\Theta} K(x, y) w(t, y) \mathrm{d} y
$$

The kernel $K(x, y)$ is equal to $\nabla_{x}^{\perp} G(x, y)=\left(-\partial_{x_{2}} G(x, y), \partial_{x_{1}} G(x, y)\right)$, where $G(x, y)$ is the fundamental solution of the Poisson equation

$$
\begin{aligned}
\Delta_{x} G(x, y) & =\delta_{y}(x), & & x \in \Theta, \\
G(x, y) & =0, & & x \in \partial \Theta .
\end{aligned}
$$

Let us remark on the important properties of the kernel $K$ :

$$
\begin{aligned}
& \left.\forall(x, y) \in \bar{\Theta}^{2} \quad \text { with } x \neq y, \quad \nabla_{x} \cdot K(x, y)=0,\right\} \\
& \forall(x, y) \in \partial \Theta \times \bar{\Theta} \quad \text { with } x \neq y, \quad K(x, y) \cdot n(x)=0 .\}
\end{aligned}
$$

Throughout the rest of the paper we will moreover make the following assumptions.

Hypotheses (H). The domain $\Theta$ of $\mathbb{R}^{2}$ is bounded, simply connected and of class $\mathcal{C}^{4}$. We assume that $w_{0}$ and $g$ are non-zero functions, and that

$$
w_{0} \in L^{2}(\Theta), \quad g(t, x) \in L_{t}^{2}\left([0, T], L_{x}^{2}(\partial \Theta, \mathrm{d} \sigma)\right) .
$$

where $\mathrm{d} \sigma(x)$ denotes the surface measure on the boundary.

We recall that the domain $\Theta$ of class $\mathcal{C}^{4}$ satisfies the uniform 'exterior sphere' condition:

$$
\exists C_{\mathrm{sp}} \geqslant 0, \quad \forall x \in \partial \Theta, \quad \forall x^{\prime} \in \bar{\Theta}, \quad C_{\mathrm{sp}}\left|x-x^{\prime}\right|^{2}+n(x) \cdot\left(x-x^{\prime}\right) \geqslant 0 .
$$


We further denote by $H$ a $\mathcal{C}_{b}^{2}(\bar{\Theta})$-extension of the distance function $d(\cdot, \partial \Theta)$ (defined on a restriction to $\Theta$ of a neighbourhood of $\partial \Theta$ ). The function $H$ satisfies (see [11])

$$
\nabla H=-n \text { on } \partial \Theta .
$$

Thanks to the assumptions made on $\Theta$, the following properties hold for the Green function $G$ and the kernel $K=\left(K_{1}, K_{2}\right)$.

Lemma 2.1. $\exists C_{0}>0, \forall x \neq y \in \bar{\Theta}$,

$$
\begin{gathered}
|G(x, y)| \leqslant C_{0}(1+|\ln | x-y||), \quad|K(x, y)| \leqslant \frac{C_{0}}{|x-y|}, \\
\left|\nabla_{x} K_{i}(x, y)\right|+\left|\nabla_{y} K_{i}(x, y)\right| \leqslant \frac{C_{0}}{|x-y|^{2}} \quad \text { for } i=1,2 .
\end{gathered}
$$

Proof. For $y=\left(y_{1}, y_{2}\right) \in \mathbb{R}^{2}$, let $y^{\perp}=\left(-y_{2}, y_{1}\right)$ and $y^{*}=y /|y|^{2}$ if $y \neq(0,0)$.

In the case in which $\Theta$ is the unit disk $B(0,1)$ of $\mathbb{R}^{2}$, one has the following explicit expression for the Green function (see [11, p. 19]):

$$
G_{0}(x, y)=\frac{1}{2 \pi} \ln \left(\frac{|x-y|}{|y|\left|x-y^{*}\right|}\right) .
$$

We remark that

$$
\forall x, y \in \bar{B}(0,1), \quad\left|x-y^{*}\right| \geqslant|y|\left|x-y^{*}\right|=\sqrt{|x-y|^{2}+\left(|x|^{2}-1\right)\left(|y|^{2}-1\right)} \geqslant|x-y| .
$$

As a consequence,

$$
\left|2 \pi G_{0}(x, y)\right| \leqslant-\ln |x-y| 1_{\{|x-y| \leqslant 1\}}+\ln \left(|y|\left|x-y^{*}\right|\right) 1_{\left\{|y|\left|x-y^{*}\right| \geqslant 1\right\}} .
$$

As $|y|\left|x-y^{*}\right|=|x| y|-y /| y|| \leqslant 2$, we conclude that $\left|2 \pi G_{0}(x, y)\right| \leqslant|\ln | x-y||+\ln (2)$.

We also deduce from (2.9) the bound on the corresponding kernel:

$$
K(x, y)=\frac{1}{2 \pi}\left(\frac{(x-y)^{\perp}}{|x-y|^{2}}-\frac{\left(x-y^{*}\right)^{\perp}}{\left|x-y^{*}\right|^{2}}\right)=\frac{1}{2 \pi}\left(\left((x-y)^{*}\right)^{\perp}-\left(\left(x-y^{*}\right)^{*}\right)^{\perp}\right) .
$$

To estimate $\nabla K_{i}$, we combine (2.9) and the fact that each term of the Jacobian matrix of $z \rightarrow z^{*}$ is bounded by $1 /|z|^{2}$.

When $\Theta$ is a general bounded and simply connected domain of class $\mathcal{C}^{3}$, according to [21], there is a conformal mapping from $B(0,1)$ onto $\Theta$ which extends to a one-to-one $\mathcal{C}^{2}$ mapping from $\bar{B}(0,1)$ to $\bar{\Theta}$ denoted by $f$ and such that $D f,(D f)^{-1}$ and $D^{2} f$ are bounded on $\bar{B}(0,1)$. Since the Green function for $\Theta$ is given by

$$
G(x, y)=G_{0}\left(f^{-1}(x), f^{-1}(y)\right),
$$

the estimations on $G, K$ and $\nabla K_{i}$ follow from those obtained for the unit disk and the mentioned properties of $f$. 
We are interested in weak solutions of (2.1) defined in the following sense.

Definition 2.2. We say that $w:[0, T] \times \Theta \rightarrow \mathbb{R}$ is a weak solution of (2.1) if

(i) $w \in L_{t}^{\infty}\left(L_{x}^{2}\right) \cap L_{t}^{2}\left(H_{x}^{1}\right)$, where $L_{t}^{\infty}\left(L_{x}^{2}\right)$ and $L_{t}^{2}\left(H_{x}^{1}\right)$ stand, respectively, for $L^{\infty}\left([0, T], L^{2}(\Theta)\right)$ and $L^{2}\left([0, T], H^{1}(\Theta)\right)$ (and $H^{1}(\Theta)$ is the Sobolev space consisting of functions which belong together with their first-order distribution derivatives to $\left.L^{2}(\Theta)\right)$;

(ii) for any $v \in H^{1}(\Theta)$,

$$
\frac{\mathrm{d}}{\mathrm{d} t} \int_{\Theta} w_{t} v+\nu \int_{\Theta} \nabla w_{t} \cdot \nabla v=\int_{\Theta} w_{t} K w_{t} \cdot \nabla v+\nu \int_{\partial \Theta} g_{t} v \mathrm{~d} \sigma
$$

holds in $\mathcal{D}^{\prime}(] 0, T[)$;

(iii) $w(0, \cdot)=w_{0}$.

Remark 2.3. The variational formulation (ii) is well defined. Indeed, by the trace theory (see [5, pp. 196, 197]),

$$
\forall v \in H^{1}(\Theta), \quad\left|\int_{\partial \Theta} g_{t} v \mathrm{~d} \sigma\right| \leqslant C\left\|g_{t}\right\|_{L^{2}(\partial \Theta)}\|v\|_{H^{1}}
$$

and by Hypotheses $(\mathrm{H})$, the second term of the right-hand side belongs to $L_{t}^{2}$. In addition, according to Lemma 2.4 below,

$$
\forall v \in H^{1}(\Theta), \quad\left|\int_{\Theta} w_{t} K w_{t} \cdot \nabla v\right| \leqslant C\left\|w_{t}\right\|_{L^{2}}\left\|w_{t}\right\|_{H^{1}}\|v\|_{H^{1}}
$$

and, by (i), the first term on the right-hand side also belongs to $L_{t}^{2}$. So does the second term of the left-hand side.

The above inequalities together with $\left|\int_{\Theta} \nabla w_{t} \cdot \nabla v\right| \leqslant\left\|w_{t}\right\|_{H^{1}}\|v\|_{H^{1}}$ ensure that if $w$ satisfies (i) and (ii), then the distribution derivative $\partial_{t} w$ belongs to $L_{t}^{2}\left(H_{x}^{1^{\prime}}\right)$, where $H_{x}^{1^{\prime}}$ denotes the dual space of $H^{1}(\Theta)$. Applying Lemma 1.2 on p. 261 of [25] with $H=L^{2}(\Theta)$ and $V=H^{1}(\Theta)$, we deduce that $w$ has a representative in $\mathcal{C}\left([0, T], L^{2}(\Theta)\right)$ that we still denote by $w$.

Before stating the existence of a unique weak solution to (2.1), we are going to check the following lemma which prepares us for the study of the nonlinear term in (2.1).

Lemma 2.4.

$$
\begin{gathered}
\forall 2<p \leqslant+\infty, \quad \exists C>0, \quad \forall w \in L^{p}(\Theta), \quad K w \in \mathcal{C}(\bar{\Theta}) \quad \text { and } \quad\|K w\|_{L^{\infty}} \leqslant C\|w\|_{L^{p}}, \\
\exists C>0, \quad \forall w \in L^{2}(\Theta), \quad\|K w\|_{L^{2}} \leqslant C\|w\|_{L^{2}}, \\
\exists C>0, \quad \forall u \in L^{2}(\Theta), \quad \forall v, w \in H^{1}(\Theta), \quad\left|\int_{\Theta} u K w \cdot \nabla v\right| \leqslant C\|u\|_{L^{2}}\|w\|_{H^{1}}\|v\|_{H^{1}} \\
\forall u, v, w \in H^{1}(\Theta), \quad \int_{\Theta} v K w \cdot \nabla v=0 \quad \text { and } \quad \int_{\Theta} u K w \cdot \nabla v=-\int_{\Theta} v K w \cdot \nabla u
\end{gathered}
$$


Proof. For $\alpha>0$, let $K_{\alpha}(x, y)=\mathbf{1}_{\{|x-y|>\alpha\}} K(x, y)$. By Lebesgue's theorem and using the continuity of $K$ away from the diagonal, we obtain the continuity of $x \in \bar{\Theta} \mapsto K_{\alpha}(x, \cdot) \in L_{y}^{q}$, for each $q \geqslant 1$. When in addition $q<2$, according to Lemma 2.1, $K_{\alpha}(x, \cdot)$ converges to $K(x, \cdot)$ in $L_{y}^{q}$ uniformly on $\bar{\Theta}$, when $\alpha$ tends to 0 . We deduce that $K(x, \cdot)$ is continuous in $L_{y}^{q}$ and obtain $(2.11)$ by Hölder's inequality.

Let $w \in L^{2}(\Theta)$. Using Lemma 2.1 and the Cauchy-Schwarz inequality, we get

$$
\|K w\|_{L^{2}}^{2} \leqslant \int_{\Theta}\left(\int_{\Theta} \frac{C_{0}}{|x-y|} \mathrm{d} y\right)\left(\int_{\Theta} \frac{C_{0} w^{2}(y)}{|x-y|} \mathrm{d} y\right) \mathrm{d} x \leqslant\left(\sup _{x \in \bar{\Theta}} \int_{\Theta} \frac{C_{0}}{|x-y|} \mathrm{d} y\right)^{2}\|w\|_{L^{2}}^{2} .
$$

Combining, for $2<p<+\infty,(2.11)$ and the Sobolev inequality $\|w\|_{L^{p}} \leqslant C\|w\|_{H^{1}}[\mathbf{5}$, p. 165], we get

$$
\|K w\|_{L^{\infty}} \leqslant C\|w\|_{H^{1}}
$$

and conclude that (2.13) holds by the Cauchy-Schwarz inequality.

We deduce that $v, w \in H^{1}(\Theta) \rightarrow \int_{\Theta} v K w \cdot \nabla v$ is continuous. Since, according to [5, p. 162], the restrictions to $\Theta$ of $\mathcal{C}^{\infty}$ functions with compact support on $\mathbb{R}^{2}$ are dense in $H^{1}(\Theta)$, it is enough to check the first equality in (2.14) for smooth $v, w$. For $\alpha>0$, let

$$
G_{\alpha} w(x)=\int_{\Theta} \mathbf{1}_{\{|x-y|>\alpha\}} G(x, y) w(y) \mathrm{d} y .
$$

By Lemma 2.1, $K_{\alpha} w(x)=\nabla^{\perp}\left(G_{\alpha} w\right)(x)$ and $G_{\alpha} w$ and $K_{\alpha} w$ converge uniformly on $\bar{\Theta}$, respectively, to $G w$ and $K w$. Since $K w$ is continuous, we deduce that $K w=\nabla^{\perp}(G w)$ and $\nabla \cdot K w=0$. The boundary condition $\forall x \in \partial \Theta, G w(x)=0$ implies that the tangential derivative of $G w$ vanishes on $\partial \Theta$, i.e. $\forall x \in \partial \Theta, K w(x) \cdot n(x)=0$. Using Green's formula we deduce

$$
\int_{\Theta} v K w \cdot \nabla v=\frac{1}{2} \int_{\Theta} K w \cdot \nabla v^{2}=\frac{1}{2} \int_{\partial \Theta} v^{2} K w \cdot n \mathrm{~d} \sigma-\frac{1}{2} \int_{\Theta} v^{2} \nabla \cdot K w=0 .
$$

The second equality in (2.14) is deduced by polarization.

\section{Remark 2.5.}

(1) According to [5, p. 195], one also gets

$$
\forall w \in H^{1}(\Theta), \quad\|w\|_{L^{4}} \leqslant C\|w\|_{L^{2}}^{1 / 2}\|w\|_{H^{1}}^{1 / 2},
$$

and one deduces that any weak solution of (2.1) belongs to $L_{t}^{4}\left(L_{x}^{4}\right)$.

(2) For $w \in H^{1}(\Theta)$, since $G w$ solves $\Delta G w=w$ with homogeneous Dirichlet boundary conditions, by [5, Theorem IX.25, p. 181], $G w \in H^{3}(\Theta)$ and $\|G w\|_{H^{3}} \leqslant C\|w\|_{H^{1}}$. Since we have just seen above that $K w=\nabla^{\perp}(G w)$, we deduce that $K w \in\left(H^{2}(\Theta)\right)^{2}$ with $\|K w\|_{\left(H^{2}\right)^{2}} \leqslant C\|w\|_{H^{1}}$. Then, using Theorem 2 in Zuazua [27] and (2.15), we deduce that

$$
\forall x, y \in \bar{\Theta}, \quad|K w(x)-K w(y)| \leqslant C\|w\|_{H^{1}} \phi\left(\frac{|x-y|}{d(\Theta)}\right),
$$


where $d(\Theta)$ denotes the diameter of $\Theta$ and, for $r \geqslant 0, \phi(r)=(r \wedge 1)(1-\log (r \wedge 1))$. The function $\phi$ is non-decreasing and concave on $\mathbb{R}_{+}$.

We are now ready to prove the following theorem.

Theorem 2.6. Under Hypotheses (H), Equation (2.1) has a unique solution $w$ in the sense of Definition 2.2. In addition, $w \in C\left([0, T], L_{x}^{2}\right) \cap L_{t}^{4}\left(L_{x}^{4}\right)$.

Proof. The last assertion is a consequence of Remark 2.5.

Uniqueness. The proof is similar to the one made for the two-dimensional NavierStokes equation (see, for instance, [25, p. 294]).

Let $v$ and $w$ denote two solutions and $\tilde{w}=v-w$. As $\tilde{w} \in L_{t}^{2}\left(H_{x}^{1}\right)$ and by Remark 2.3, $\partial_{t} \tilde{w} \in L_{t}^{2}\left(H_{x}^{1^{\prime}}\right)$, according to $\left[\mathbf{2 5}\right.$, p. 261], $(\mathrm{d} / \mathrm{d} t)\|\tilde{w}(t)\|_{L^{2}}^{2}=2\left\langle\partial_{t} \tilde{w}_{t}, \tilde{w}_{t}\right\rangle$ holds in the distribution sense on $[0, T]$. The right-hand side is integrable. Using Definition 2.2 (ii) and (iii), we deduce that for $t \in[0, T]$,

$$
\frac{1}{2}\|\tilde{w}(t)\|_{L^{2}}^{2}+\nu \int_{0}^{t}\left\|\nabla \tilde{w}_{s}\right\|_{L^{2}}^{2} \mathrm{~d} s=\int_{0}^{t} \int_{\Theta}\left(v_{s} K v_{s}-w_{s} K w_{s}\right) \cdot \nabla \tilde{w}_{s} \mathrm{~d} s .
$$

Using (2.14) and then (2.13) and Young's inequality, we have

$$
\begin{aligned}
\left|\int_{\Theta}\left(v_{s} K v_{s}-w_{s} K w_{s}\right) \cdot \nabla \tilde{w}_{s}\right| & =\left|\int_{\Theta} \tilde{w}_{s} K v_{s} \cdot \nabla \tilde{w}_{s}+w_{s} \cdot K \tilde{w}_{s} \cdot \nabla \tilde{w}_{s}\right| \\
& =\left|0-\int_{\Theta} \tilde{w}_{s} K \tilde{w}_{s} \cdot \nabla w_{s}\right| \\
& \leqslant C\left\|\tilde{w}_{s}\right\|_{H^{1}}\left\|w_{s}\right\|_{H^{1}}\left\|\tilde{w}_{s}\right\|_{L^{2}} \\
& \leqslant \nu\left(\|\nabla \tilde{w}(s)\|_{L^{2}}^{2}+\|\tilde{w}(s)\|_{L^{2}}^{2}\right)+\frac{C^{2}}{4 \nu}\left\|w_{s}\right\|_{H^{1}}^{2}\left\|\tilde{w}_{s}\right\|_{L^{2}}^{2} .
\end{aligned}
$$

Inserting this bound in (2.18), we obtain

$$
\forall t \in[0, T], \quad\|\tilde{w}(t)\|_{L^{2}}^{2} \leqslant 2 \int_{0}^{t}\left(\nu+\frac{C^{2}}{4 \nu}\left\|w_{s}\right\|_{H^{1}}^{2}\right)\left\|\tilde{w}_{s}\right\|_{L^{2}}^{2} \mathrm{~d} s .
$$

Since $s \rightarrow\left\|w_{s}\right\|_{H^{1}}^{2}$ is integrable, by Gronwall's lemma, $\forall t \in[0, T],\left\|v_{t}-w_{t}\right\|_{L^{2}}=0$.

Existence. Let us apply the Galerkin method. We first derive an a priori estimate which will also hold at the discrete level. Let $w$ be a weak solution of (2.1). As above,

$$
\frac{1}{2} \frac{\mathrm{d}}{\mathrm{d} t}\left\|w_{t}\right\|_{L^{2}}^{2}+\nu\left\|\nabla w_{t}\right\|_{L^{2}}^{2}=\int_{\Theta} w_{t} K w_{t} \cdot \nabla w_{t}+\nu \int_{\partial \Theta} g_{t} w_{t} \mathrm{~d} \sigma .
$$

According to (2.14), the first term on the right-hand side is zero. Using, moreover, (2.10) and Young's inequality, we deduce

$$
\frac{1}{2} \frac{\mathrm{d}}{\mathrm{d} t}\left\|w_{t}\right\|_{L^{2}}^{2}+\nu\left\|\nabla w_{t}\right\|_{L^{2}}^{2} \leqslant \frac{1}{2} \nu\left(\left\|\nabla w_{t}\right\|_{L^{2}}^{2}+\left\|w_{t}\right\|_{L^{2}}^{2}\right)+C\left\|g_{t}\right\|_{L^{2}(\partial \Theta)}^{2} .
$$


Removing the terms involving $\left\|\nabla w_{t}\right\|_{L^{2}}^{2}$, we upper bound $\|w\|_{L_{t}^{\infty}\left(L_{x}^{2}\right)}^{2}$ by Gronwall's lemma. Inserting this bound in (2.19), we conclude that

$$
\|w\|_{L_{t}^{\infty}\left(L_{x}^{2}\right)}^{2}+\|\nabla w\|_{L_{t}^{2}\left(L_{x}^{2}\right)}^{2} \leqslant C_{T}\left(\left\|w_{0}\right\|_{L^{2}}^{2}+\|g\|_{L_{t}^{2}\left(L_{x}^{2}(\partial \Theta)\right)}^{2}\right) .
$$

We now employ the so-called Galerkin method. Adapting [5, pp. 192, 193] to diagonalize the Neumann Laplacian, we obtain a Hilbertian basis $\left(u_{k}\right)_{k \in \mathbb{N}^{*}}$ of $L^{2}(\Theta)$. These functions are such that

$$
\begin{array}{r}
\forall k, \quad \exists \mu_{k} \geqslant 0, \quad \forall v \in H^{1}(\Theta), \quad \int_{\Theta} \nabla v \cdot \nabla u_{k}=\mu_{k} \int_{\Theta} v u_{k}, \\
\text { i.e. }\left(v, u_{k}\right)_{H^{1}}=\left(1+\mu_{k}\right)\left(v, u_{k}\right)_{L^{2}} .
\end{array}
$$

As a consequence, the functions $v_{k}=u_{k} / \sqrt{1+\mu_{k}}, k \geqslant 1$, form a Hilbertian basis of $H^{1}(\Theta)$.

We want to find $t \in[0, T] \rightarrow \Lambda(t)=\left(\lambda_{1}(t), \ldots, \lambda_{n}(t)\right)$ such that $w_{t}^{n}=\sum_{k=1}^{n} \lambda_{k}(t) v_{k}$ satisfies the following approximate problem: $w_{0}^{n}$ is the orthogonal projection in the sense of the $L^{2}$ scalar product of $w_{0}$ onto $\operatorname{span}\left(v_{1}, \ldots, v_{n}\right)$ and

$$
\forall 1 \leqslant k \leqslant n, \quad \frac{\mathrm{d}}{\mathrm{d} t} \int_{\Theta} w_{t}^{n} v_{k}+\nu \int_{\Theta} \nabla w_{t}^{n} \cdot \nabla v_{k}=\int_{\Theta} w_{t}^{n} K w_{t}^{n} \cdot \nabla v_{k}+\nu \int_{\partial \Theta} g_{t} v_{k} \mathrm{~d} \sigma .
$$

Denoting

$$
\begin{gathered}
A_{j k}=\int_{\Theta} v_{j} v_{k}, \quad B_{j, k}=\int_{\Theta} \nabla v_{j} \cdot \nabla v_{k} \\
C_{i, j, k}=\int_{\Theta} v_{i} K v_{j} \cdot \nabla v_{k}, \quad \Gamma(t)=\left(\gamma_{1}(t), \ldots, \gamma_{n}(t)\right) \quad \text { with } \gamma_{k}(t)=\int_{\partial \Theta} g_{t} v_{k} \mathrm{~d} \sigma,
\end{gathered}
$$

we obtain that this approximate problem can be written as

$$
\frac{\mathrm{d}}{\mathrm{d} t} \Lambda(t)=A^{-1}\left(-\nu B \cdot \Lambda(t)+\Lambda(t)^{*} C \Lambda(t)+\nu \Gamma(t)\right) .
$$

By a standard fixed-point approach, we obtain existence of a local-in-time solution $\Lambda(t)$ to this ordinary differential equation. Thanks to the a priori estimate $(2.20)$ which holds for the corresponding $w_{t}^{n}$, and prevents explosion for $\Lambda(t)$, we can iterate this fixed-point approach to extend $\Lambda(t)$ on $[0, T]$.

We next want to take the limit $n \rightarrow+\infty$. According to the a priori estimate (2.20), the sequence $\left(w^{n}\right)_{n \in \mathbb{N}^{*}}$ is bounded in $L_{t}^{\infty}\left(L_{x}^{2}\right)$ and $L_{t}^{2}\left(H_{x}^{1}\right)$. Hence we may extract a subsequence that converges to a limit $w$ weakly* in $L_{t}^{\infty}\left(H_{x}^{1}\right)$ and weakly in $L_{t}^{2}\left(H_{x}^{1}\right)$. The weak and weak ${ }^{*}$ convergences of these subsequences are enough to take the limit in the linear terms of (2.22) (see [25, pp. 257-260]).

In order to take the limit in the nonlinear term, we need a stronger convergence. Since the functions $u_{k}=\sqrt{1+\mu_{k}} v_{k}$ form a Hilbertian basis of $L^{2}(\Theta)$, one obtains, using (2.21),

$$
\forall v \in H^{1}(\Theta), \quad \int_{\Theta} w_{t}^{n} v=\int_{\Theta} w_{t}^{n} \sum_{k=1}^{n}\left(v, u_{k}\right)_{L^{2}} u_{k}=\int_{\Theta} w_{t}^{n} \sum_{k=1}^{n}\left(v, v_{k}\right)_{H^{1}} v_{k} .
$$


Therefore, by (2.22),

$$
\begin{aligned}
\frac{\mathrm{d}}{\mathrm{d} t} \int_{\Theta} w_{t}^{n} v+\nu \int_{\Theta} \nabla w_{t}^{n} & \cdot \sum_{k=1}^{n}\left(v, v_{k}\right)_{H^{1}} \nabla v_{k} \\
& =\int_{\Theta} w_{t}^{n} K w_{t}^{n} \cdot \nabla \sum_{k=1}^{n}\left(v, v_{k}\right)_{H^{1}} v_{k}+\nu \sum_{k=1}^{n}\left(v, v_{k}\right)_{H^{1}} \int_{\partial \Theta} g_{t} v_{k} \mathrm{~d} \sigma
\end{aligned}
$$

Since $\left\|\sum_{k=1}^{n}\left(v, v_{k}\right)_{H^{1}} v_{k}\right\|_{H^{1}} \leqslant\|v\|_{H^{1}}$, reasoning as in Remark 2.3, we check that $\left(\partial_{t} w^{n}\right)_{n \in \mathbb{N}^{*}}$ is bounded in $L_{t}^{2}\left(H_{x}^{1^{\prime}}\right)$. Let $2<p<+\infty$. Since $H^{1}(\Theta) \hookrightarrow L^{p}(\Theta) \hookrightarrow\left(H^{1}(\Theta)\right)^{\prime}$ and the first of these injections is compact (see [5, Theorem IX.16, p. 169]), according to $[\mathbf{1 5}$, Theorem 5.1, p. 58], we deduce that we may extract a further subsequence that converges to $w$ strongly in $L_{t}^{2}\left(L_{x}^{p}\right)$. This subsequence is still denoted by $\left(w^{n}\right)$ for notational simplicity. By (2.11) and the strong convergence in $L_{t}^{2}\left(L_{x}^{p}\right), w_{t}^{n} K w_{t}^{n}$ converges to $w_{t} K w_{t}$ strongly in $L_{t}^{1}\left(L_{x}^{p}\right)$. Therefore,

$$
\forall \psi \in \mathcal{D}(] 0, T[), \quad \int_{0}^{T} \psi^{\prime}(t) \int_{\Theta} w_{t}^{n} K w_{t}^{n} \cdot \nabla v \mathrm{~d} t \rightarrow \int_{0}^{T} \psi^{\prime}(t) \int_{\Theta} w_{t} K w_{t} \cdot \nabla v \mathrm{~d} t .
$$

Hence $w$ satisfies (ii) in Definition 2.2. Since, by standard arguments [25, pp. 257-260], (iii) also holds, we conclude that $w$ is a weak solution of (2.1).

In order to give a probabilistic interpretation of the obtained weak solution of (2.1), we introduce the semi-group $P_{t}^{\nu}(x, y)$ associated with $\sqrt{2 \nu}$ times the Brownian motion normally reflected on the boundary and prove the following mild representation.

Proposition 2.7. Let $w$ denote the weak solution of (2.1) given by Theorem 2.6. Then $\forall t \in[0, T], \mathrm{d} x$ a.e. in $\Theta$,

$$
w_{t}(x)=P_{t}^{\nu} w_{0}(x)+\int_{0}^{t} \nabla P_{t-s}^{\nu} \cdot\left(w_{s} K w_{s}\right)(x) \mathrm{d} s+\nu \int_{0}^{t} \int_{\partial \Theta} P_{t-s}^{\nu}(y, x) g(s, y) \mathrm{d} \sigma(y) \mathrm{d} s,
$$

where

$$
\nabla P_{t-s}^{\nu} \cdot\left(w_{s} K w_{s}\right)(x)=\int_{\Theta} \nabla_{y} P_{t-s}^{\nu}(y, x) \cdot w_{s}(y) K w_{s}(y) \mathrm{d} y .
$$

Proof. Let $t \in] 0, T]$ and let $\varphi$ be a smooth function on $\bar{\Theta}$ with a vanishing normal derivative at the boundary: $\partial_{n} \varphi(x)=0$ for $x \in \partial \Theta$. According to [14, Theorem 5.3, p. 320], the boundary-value problem

$$
\begin{aligned}
\partial_{s} \psi+\nu \Delta \psi & =0 & & \text { on }[0, t] \times \Theta, \\
\partial_{n} \psi & =0 & & \text { on }[0, t] \times \partial \Theta, \\
\psi(t, \cdot) & =\varphi(\cdot) & & \text { on } \Theta
\end{aligned}
$$

admits a classical solution $\psi(s, x)$ which is $\mathcal{C}^{1,2}$ on $[0, t] \times \bar{\Theta}$. By the Feynman-Kac approach, this solution has the following representation: $\psi(s, x)=P_{t-s}^{\nu} \varphi(x)$. Clearly, 
$\psi \in L^{\infty}\left([0, t], H^{1}(\Theta)\right)$ and $\partial_{s} \psi \in L^{2}\left([0, t],\left(H^{1}\right)_{x}^{\prime}(\Theta)\right)$. By [25, Lemma 1.2, p. 261] we deduce that in $\mathcal{D}^{\prime}(] 0, t[)$,

$$
\begin{array}{r}
\frac{\mathrm{d}}{\mathrm{d} s} \int_{\Theta} w_{s} \psi(s, \cdot)=\int_{\Theta} w_{s} \partial_{s} \psi(s, \cdot)-\nu \int_{\Theta} \nabla w_{s} \cdot \nabla \psi(s, \cdot)+\int_{\Theta} w_{s} K w_{s} \cdot \nabla \psi(s, \cdot) \\
+\nu \int_{\partial \Theta} g_{s} \psi(s, \cdot) \mathrm{d} \sigma .
\end{array}
$$

By the equation satisfied by $\psi$, the sum of the two first terms on the right-hand side is zero. Hence

$$
\begin{aligned}
\int_{\Theta} w_{t}(x) \varphi(x) \mathrm{d} x=\int_{\Theta} w_{0}(x) \psi(0, x) \mathrm{d} x+\int_{0}^{t} \int_{\Theta} & w_{s} K w_{s}(x) \cdot \nabla \psi(s, x) \mathrm{d} x \mathrm{~d} s \\
& +\nu \int_{0}^{t} \int_{\partial \Theta} \psi(s, x) g(s, x) \mathrm{d} \sigma(x) \mathrm{d} s .
\end{aligned}
$$

By Hypotheses (H),

$$
\int_{0}^{t} \int_{\partial \Theta} \int_{\Theta} P_{t-s}^{\nu}(x, y)|\varphi(y)| \mathrm{d} y|g(s, x)| \mathrm{d} \sigma(x) \mathrm{d} s \leqslant\|\varphi\|_{L^{\infty}}\|g\|_{L_{t}^{1}\left(L_{x}^{1}(\partial \Theta)\right)}<+\infty .
$$

Hence, by Fubini's theorem the last term on the right-hand side is equal to

$$
\nu \int_{\Theta} \varphi(x) \int_{0}^{t} \int_{\partial \Theta} P_{t-s}^{\nu}(y, x) g(s, y) \mathrm{d} \sigma(y) \mathrm{d} s \mathrm{~d} x .
$$

We conclude the proof by applying, similarly, Fubini's theorem to the other terms on the right-hand side and remarking that the derived equality holds for any smooth function $\varphi$ with vanishing normal derivative.

To justify the use of Fubini's theorem in the second term, we need the following estimations given by $[\mathbf{2 2},($ a.13), (a.14), p. 600]:

$$
\forall x \in \bar{\Theta}, \quad \forall y \in \bar{\Theta}, \quad\left|\nabla_{x} P_{t}^{\nu}(x, y)\right| \leqslant C_{1} / t^{3 / 2} \quad \text { and } \quad\left\|\nabla_{x} P_{t}^{\nu}(x, y)\right\|_{L_{y}^{1}(\Theta)} \leqslant C_{1} / \sqrt{t}
$$

Indeed the first one ensures that $\nabla \psi(s, x)=\int_{\Theta} \nabla_{x} P_{t-s}^{\nu}(x, y) \varphi(y) \mathrm{d} y$. By the second one and (2.12),

$$
\begin{aligned}
& \int_{0}^{t} \int_{\Theta}\left|w_{s} K w_{s}\right|(x) \int_{\Theta}\left|\nabla_{x} P_{t-s}^{\nu}(x, y)\right||\varphi(y)| \mathrm{d} y \mathrm{~d} x \mathrm{~d} s \\
& \qquad C\|\varphi\|_{L^{\infty}}\|w\|_{L_{t}^{\infty}\left(L_{x}^{2}\right)}^{2} \int_{0}^{t}(t-s)^{-1 / 2} \mathrm{~d} s .
\end{aligned}
$$




\section{The probabilistic interpretation of the vortex equation on a bounded domain with a Neumann boundary condition}

We are in a McKean-Vlasov context, and the interpretation of the vortex equation as a Fokker-Planck equation allows us to associate naturally with this equation a nonlinear stochastic differential equation (see, for example, [18]).

Here the difficulty is the treatment of the term due to the Neumann condition involving the function $g$. We essentially follow Fernandez and Méléard [9] and prove that this term is related to space-time random births located at the boundary in the probabilistic interpretation. Our situation is harder than the one of $[\mathbf{9}]$ since we are in a bounded domain instead of the whole space and the diffusion processes are reflected on the boundary. There are also births inside the domain at time 0 and the functions $w_{0}$ and $g$ are not probability densities.

We follow Jourdain $[\mathbf{1 3}]$ to treat the last difficulty.

Let

$$
\left\|w_{0}\right\|_{1}=\int_{\Theta}\left|w_{0}\right| \quad \text { and } \quad\|g\|_{1}=\int_{[0, T] \times \partial \Theta}|g| \mathrm{d} \sigma \mathrm{d} t .
$$

To govern the times and positions of births, we introduce on $[0, T] \times \bar{\Theta}$ the probability measure

$$
P_{0}(\mathrm{~d} t, \mathrm{~d} x)=\mathbf{1}_{\{x \in \Theta\}} \delta_{\{0\}}(\mathrm{d} t) \frac{\left|w_{0}(x)\right|}{\left\|w_{0}\right\|_{1}+\nu\|g\|_{1}} \mathrm{~d} x+\mathbf{1}_{\{x \in \partial \Theta\}} \frac{\nu|g(t, x)|}{\left\|w_{0}\right\|_{1}+\nu\|g\|_{1}} \mathrm{~d} t \mathrm{~d} \sigma(x),
$$

which does not weight $] 0, T] \times \Theta$. To take into account the effect of the sign and mass of $w_{0}$ and $g$, we also consider for $t \in[0, T]$ and $x \in \bar{\Theta}$ the measurable function

$$
h(t, x)=\left(\left\|w_{0}\right\|_{1}+\nu\|g\|_{1}\right)\left(\mathbf{1}_{\{t=0, x \in \Theta\}} \frac{w_{0}(x)}{\left|w_{0}(x)\right|}+\mathbf{1}_{\{x \in \partial \Theta\}} \frac{g(t, x)}{|g(t, x)|}\right)
$$

with values in $\left\{-\left(\left\|w_{0}\right\|_{1}+\nu\|g\|_{1}\right), 0,\left\|w_{0}\right\|_{1}+\nu\|g\|_{1}\right\}$. Let us remark that if $\varphi$ is a bounded measurable function on $[0, T] \times \bar{\Theta}$, then

$$
\begin{aligned}
\int_{[0, T] \times \bar{\Theta}} \varphi(t, x) h(t, x) & P_{0}(\mathrm{~d} t, \mathrm{~d} x) \\
= & \int_{\Theta} \varphi(0, x) w_{0}(x) \mathrm{d} x+\nu \int_{[0, T] \times \partial \Theta} \varphi(t, x) g(t, x) \mathrm{d} t \mathrm{~d} \sigma(x) .
\end{aligned}
$$

We are now going to give the probabilistic interpretation of the vortex equation. This interpretation is inspired from Sznitman [23] and Bossy and Jourdain [4] for the reflected contribution and from Fernandez and Méléard [9] for the space-time random-birth contribution.

Let us first define the probability space in which the law of the solution of the stochastic differential equation will live. 
Definition 3.1. Let $\left(\tau,\left(X_{t}\right)_{t \leqslant T},\left(k_{t}\right)_{t \leqslant T}\right)$ denote the canonical process on $[0, T] \times$ $\mathcal{C}([0, T], \bar{\Theta}) \times \mathcal{C}\left([0, T], \mathbb{R}^{2}\right)$. We denote by $\mathcal{P}_{T}$ the space of probability measures $P$ on this space such that for each $t \in[0, T]$, the signed measure $\tilde{P}_{t}$ defined by

$$
\forall B \in \mathcal{B}(\bar{\Theta}), \quad \tilde{P}_{t}(B)=E^{P}\left(h\left(\tau, X_{0}\right) \mathbf{1}_{\{\tau \leqslant t\}} \mathbf{1}_{B}\left(X_{t}\right)\right),
$$

has a density $\tilde{p}_{t}$ with respect to the Lebesgue measure and $t \in[0, T] \rightarrow \tilde{p}_{t} \in L_{t}^{\infty}\left(L_{x}^{2}\right) \cap$ $L_{t}^{2}\left(H_{x}^{1}\right)$.

Definition 3.2. We denote by $(\mathbf{E})$ the following nonlinear stochastic differential equation with reflection: given an $\mathbb{R}^{2}$-valued Brownian motion $B$ and random variables $\left(\tau, X_{0}\right)$ with values in $[0, T] \times \bar{\Theta}$ and distributed according to $P_{0}$, a process $\left(\tau,\left(X_{t}\right)_{t \leqslant T},\left(k_{t}\right)_{t \leqslant T}\right)$ is a solution of $(\mathbf{E})$ if

the law $P$ of $(\tau, X, k)$ belongs to $\mathcal{P}_{T}$,

$$
\begin{array}{r}
X_{t}=X_{0}+\sqrt{2 \nu} \int_{0}^{t} \mathbf{1}_{\{\tau \leqslant s\}} \mathrm{d} B_{s}+\int_{0}^{t} \mathbf{1}_{\{\tau \leqslant s\}} K \tilde{p}_{s}\left(X_{s}\right) \mathrm{d} s-k_{t}, \\
|k|_{t}=\int_{0}^{t} \mathbf{1}_{\left\{X_{s} \in \partial \Theta\right\}} \mathbf{1}_{\{\tau \leqslant s\}} \mathrm{d}|k|_{s}, \quad k_{t}=\int_{0}^{t} n\left(X_{s}\right) \mathrm{d}|k|_{s},
\end{array}
$$

where $t \in[0, T] \rightarrow|k|_{t}$ denotes the variation function associated with $t \in[0, T] \rightarrow k_{t}$.

Note that as $P \in \mathcal{P}_{T}, P$ a.s., $\forall t \in[0, T], X_{t} \in \bar{\Theta}$. Moreover, the drift coefficient $K \tilde{p}_{s}$ is nonlinear since it depends on the density $\tilde{p}_{s}$ of the signed measure $\tilde{P}_{s}$.

The following lemma states the link between the nonlinear stochastic differential equation $(\mathbf{E})$ and the vortex equation (2.1).

Lemma 3.3. If $(\tau, X, k)$ is a strong solution of $(\boldsymbol{E})$, then $\tilde{p}$ is a weak solution of (2.1).

Proof. By (3.4) and (3.3), one immediately gets that $\tilde{p}_{0}=w_{0}$. Let $\psi \in \mathcal{C}^{1,2}([0, T] \times \bar{\Theta})$ be such that $\forall(s, x) \in[0, T] \times \partial \Theta, \partial_{n} \psi(s, x)=0$. By Itô's formula,

$$
\begin{aligned}
\psi\left(T, X_{t}\right)=\psi(\tau, & \left.X_{0}\right)+\sqrt{2 \nu} \int_{0}^{T} \mathbf{1}_{\{\tau \leqslant s\}} \nabla \psi\left(s, X_{s}\right) \cdot \mathrm{d} B_{s} \\
& +\int_{0}^{T} \mathbf{1}_{\{\tau \leqslant s\}}\left(\partial_{s} \psi\left(s, X_{s}\right)+K \tilde{p}_{s}\left(X_{s}\right) \cdot \nabla \psi\left(s, X_{s}\right)+\nu \Delta \psi\left(s, X_{s}\right)\right) \mathrm{d} s .
\end{aligned}
$$

Multiplying by $h\left(\tau, X_{0}\right)$, taking expectations and using the definition of $\tilde{p}$ and (3.3), we deduce that

$$
\begin{aligned}
\int_{\bar{\Theta}} \psi(T, x) \tilde{p}(T, x) \mathrm{d} x= & \int_{\bar{\Theta}} \psi(0, x) w_{0}(x) \mathrm{d} x+\nu \int_{0}^{T} \int_{\partial \Theta} \psi(s, x) g(s, x) \mathrm{d} \sigma(x) \mathrm{d} s \\
& +\int_{0}^{T} \int_{\bar{\Theta}}\left(\partial_{s} \psi(s, x)+K \tilde{p}_{s}(x) \cdot \nabla \psi(s, x)+\nu \Delta \psi(s, x)\right) \tilde{p}_{s}(x) \mathrm{d} x \mathrm{~d} s .
\end{aligned}
$$


For the choice $\psi(s, x)=\varphi(s) v(x)$, where $v$ is a $\mathcal{C}^{2}$ function on $\bar{\Theta}$ such that $\partial_{n} v=0$ on $\partial \Theta$ and $\varphi \in \mathcal{D}(] 0, T[)$, we obtain

$$
\int_{0}^{T}\left(\varphi^{\prime}(s) \int_{\Theta} \tilde{p}_{s} v+\varphi(s)\left(\int_{\Theta} \tilde{p}_{s} K \tilde{p}_{s} \cdot \nabla v+\nu \int_{\Theta} \tilde{p}_{s} \Delta v+\nu \int_{\partial \Theta} g_{s} v \mathrm{~d} \sigma\right)\right) \mathrm{d} s=0 .
$$

As $P \in \mathcal{P}_{T}, \tilde{p} \in L_{t}^{2}\left(H_{x}^{1}\right)$. By Green's formula for functions in $H^{1}(\Theta)[\mathbf{5}$, p. 197] and since $\partial_{n} v$ vanishes on the boundary, ds a.e. in $[0, T], \int_{\Theta} \tilde{p}_{s} \Delta v=-\int_{\Theta} \nabla \tilde{p}_{s} \cdot \nabla v$.

Since $\Theta$ is $\mathcal{C}^{4}$, the Hilbertian basis of $H^{1}(\Theta)$ introduced in the proof of Theorem 2.6 consists of $\mathcal{C}^{2}(\bar{\Theta})$ functions with a vanishing normal derivative. Therefore, such functions are dense in $H^{1}(\Theta)$ and we conclude that $\tilde{p}$ satisfies Definition 2.2 (ii).

Theorem 3.4. Under Hypotheses $(H)$, given $B$ and $\left(\tau, X_{0}\right)$, there exists a unique strong solution $(\tau, X, k)$ to the equation $(\boldsymbol{E})$. In addition, the corresponding $\tilde{p}$ is a weak solution of (2.1) and satisfies the mild equation (2.23).

Proof. We are going to check pathwise uniqueness and weak existence. The YamadaWatanabe theorem then implies existence of a strong solution.

Pathwise uniqueness. Let us consider, given $B$ and $\left(\tau, X_{0}\right)$, two strong solutions $(\tau, Z, k)$ and $(\tau, \bar{Z}, \bar{k})$ of $(\mathbf{E})$, and let us denote by $P^{1}$ the law of $(\tau, Z, k)$ and by $P^{2}$ the law of $(\tau, \bar{Z}, \bar{k})$. Then by Lemma 3.3, $\tilde{p}^{1}$ and $\tilde{p}^{2}$ are weak solutions of (2.1). According to Theorem 2.6, $\tilde{p}_{1}=\tilde{p}_{2}=w$. Hence $(\tau, Z, k)$ and $(\tau, \bar{Z}, \bar{k})$ are both solutions of the stochastic differential reflected equation $\left(E^{w}\right)$ defined like $(\mathbf{E})$, but with the known drift coefficient $K w_{s}$ replacing $K \tilde{p}_{s}$ in (3.5).

In order to prove that these two solutions are equal, let us define $h_{t}=H\left(Z_{t}\right)$, $\bar{h}_{t}=H\left(\bar{Z}_{t}\right), h_{t}^{\prime}=\nabla H\left(Z_{t}\right), \bar{h}_{t}^{\prime}=\nabla H\left(\bar{Z}_{t}\right), h_{t}^{\prime \prime}=\Delta H\left(Z_{t}\right), \bar{h}_{t}^{\prime \prime}=\Delta H\left(\bar{Z}_{t}\right)$ (where $H$ denotes the extension of the distance to the boundary introduced after the statement of Hypotheses $(\mathrm{H})), b_{t}=K w_{t}\left(Z_{t}\right)$ and $\bar{b}_{t}=K w_{t}\left(\bar{Z}_{t}\right)$. Computing $\operatorname{dexp}\left(-2 C_{\mathrm{sp}}\left(h_{t}+\bar{h}_{t}\right)\right)\left|Z_{t}-\bar{Z}_{t}\right|^{2}$ by Itô's formula, we get

$$
\begin{aligned}
& 1_{\{\tau \leqslant t\}} \exp \left(-2 C_{\mathrm{sp}}\left(h_{t}+\bar{h}_{t}\right)\right) \\
& \times\left[2\left(Z_{t}-\bar{Z}_{t}\right) \cdot\left(\mathrm{d} \bar{k}_{t}-\mathrm{d} k_{t}\right)-2 C_{\mathrm{sp}}\left|Z_{t}-\bar{Z}_{t}\right|^{2}\left(\mathrm{~d}|k|_{t}+\mathrm{d}|\bar{k}|_{t}\right)\right. \\
& \quad-2 C_{\mathrm{sp}}\left|Z_{t}-\bar{Z}_{t}\right|^{2}\left(\sqrt{2 \nu}\left(h_{t}^{\prime}+\bar{h}_{t}^{\prime}\right) \mathrm{d} B_{t}\right. \\
& \left.\quad+\left\{h_{t}^{\prime} b_{t}+\bar{h}_{t}^{\prime} \bar{b}_{t}+\nu\left(-2 C_{\mathrm{sp}}\left|h_{t}^{\prime}+\bar{h}_{t}^{\prime}\right|^{2}+h_{t}^{\prime \prime}+\bar{h}_{t}^{\prime \prime}\right)\right\} \mathrm{d} t\right) \\
& \left.+2\left(Z_{t}-\bar{Z}_{t}\right) \cdot\left(b_{t}-\bar{b}_{t}\right) \mathrm{d} t\right]
\end{aligned}
$$

Because of the 'exterior-sphere' condition (2.6), the local-time terms of the first line have a non-positive contribution after integration over time. We deduce that for a constant $C_{H}$, depending only on the upper bounds of the function $H$ and its derivatives,

$$
\begin{aligned}
E\left(\left|Z_{t}-\bar{Z}_{t}\right|^{2}\right) \leqslant C_{H}\left(\int_{0}^{t}(\|\right. & \left.K w_{s} \|_{L^{\infty}}+1\right) E\left(\left|Z_{s}-\bar{Z}_{s}\right|^{2}\right) \mathrm{d} s \\
& \left.\quad+\int_{0}^{t} E\left(\left|Z_{s}-\bar{Z}_{s}\right|\left|K w_{s}\left(Z_{s}\right)-K w_{s}\left(\bar{Z}_{s}\right)\right|\right) \mathrm{d} s\right) .
\end{aligned}
$$


By $(2.17)$ and since, for $0 \leqslant r \leqslant d(\Theta)$,

$$
\begin{gathered}
r \phi\left(\frac{r}{d(\Theta)}\right)=\frac{r^{2}}{d(\Theta)}\left(1-\log \left(\frac{r}{d(\Theta)}\right)\right) \leqslant \frac{r^{2}}{d(\Theta)}\left(1-2 \log \left(\frac{r}{d(\Theta)}\right)\right)=d(\Theta) \phi\left(\frac{r^{2}}{d(\Theta)^{2}}\right), \\
E\left(\left|Z_{s}-\bar{Z}_{s}\right|\left|K w_{s}\left(Z_{s}\right)-K w_{s}\left(\bar{Z}_{s}\right)\right|\right) \leqslant C\left\|w_{s}\right\|_{H^{1}} E\left(\phi\left(\frac{\left|Z_{s}-\bar{Z}_{s}\right|^{2}}{d(\Theta)^{2}}\right)\right) .
\end{gathered}
$$

Since the function $\phi$ is concave by Jensen's inequality,

$$
E\left(\left|Z_{s}-\bar{Z}_{s}\right|\left|K w_{s}\left(Z_{s}\right)-K w_{s}\left(\bar{Z}_{s}\right)\right|\right) \leqslant C\left\|w_{s}\right\|_{H^{1}} \phi\left(E\left(\frac{\left|Z_{s}-\bar{Z}_{s}\right|^{2}}{d(\Theta)^{2}}\right)\right) .
$$

Inserting this bound and (2.15) into (3.7), we deduce that $y(s) \stackrel{\text { def }}{=} E\left(\left|Z_{s}-\bar{Z}_{s}\right|^{2}\right) / d(\Theta)^{2}$ satisfies

$$
y(t) \leqslant C \int_{0}^{t}\left(1+\left\|w_{s}\right\|_{H^{1}}\right)(y(s)+\phi(y(s))) \mathrm{d} s .
$$

Let us introduce the ordinary differential equation

$$
z^{\prime}(t)=C\left(1+\left\|w_{t}\right\|_{H^{1}}\right)(z(t)+\phi(z(t))), \quad z(0)=\zeta>0 .
$$

Since the function $\phi$ is continuous and bounded, and $t \rightarrow\left\|w_{t}\right\|_{H^{1}}$ is integrable on $[0, T]$, by standard arguments, this equation has a non-decreasing solution on $[0, T]$ denoted by $(z(t, \zeta))_{t \in[0, T]}$. In addition,

$$
\int_{\zeta}^{z(T, \zeta)} \frac{\mathrm{d} u}{u+\phi(u)} \leqslant C \int_{0}^{T}\left(1+\left\|w_{r}\right\|_{H^{1}}\right) \mathrm{d} r<+\infty .
$$

Since

$$
\int_{0^{+}} \frac{\mathrm{d} u}{u+\phi(u)}=+\infty
$$

we deduce that

$$
z(T, \zeta)=\sup _{t \leqslant T} z(t, \zeta) \rightarrow_{\zeta \rightarrow 0} 0 .
$$

Now, since $r \rightarrow r+\phi(r)$ is increasing and Lipschitz continuous with constant $C(\zeta)$ on $[\zeta,+\infty)$, where $\zeta>0$, and $\forall t \leqslant T, z(t, \zeta) \geqslant \zeta$,

$$
\begin{aligned}
(y(t)-z(t, \zeta))^{+} & \leqslant C\left(\int_{0}^{t}\left(1+\left\|w_{s}\right\|_{H^{1}}\right)(y(s)+\phi(y(s))-(z(s, \zeta)+\phi(z(s, \zeta)))) \mathrm{d} s\right)^{+} \\
& \leqslant C \int_{0}^{t}\left(1+\left\|w_{s}\right\|_{H^{1}}\right)(y(s)+\phi(y(s))-(z(s, \zeta)+\phi(z(s, \zeta))))^{+} \mathrm{d} s \\
& \leqslant C(\zeta) \int_{0}^{t}\left(1+\left\|w_{s}\right\|_{H^{1}}\right)(y(s)-z(s, \zeta))^{+} \mathrm{d} s .
\end{aligned}
$$

By Gronwall's lemma, we deduce that $\forall t \leqslant T, y(t) \leqslant z(t, \zeta)$. Letting $\zeta \rightarrow 0$ and using (3.11), we conclude that $\forall t \leqslant T, y(t)=0$. Hence $\forall t \leqslant T, E\left(\left|Z_{t}-\bar{Z}_{t}\right|^{2}\right)=0$ and $Z_{t}=\bar{Z}_{t}$ almost surely. Since the processes are continuous, they are indistinguishable. 
Existence of a weak solution. Let $w$ be the solution of the vortex equation given by Theorem 2.6. Combining (2.11) and Remark 2.5 (1), we check that $s \mapsto\left\|K w_{s}\right\|_{L^{\infty}}$ belongs to $L^{4}([0, T])$. We will first construct a solution of the stochastic differential equation $\left(E^{w}\right)$ with known drift coefficient $K w_{s}(\cdot)$. Then we will check that its law is in fact a weak solution of $(\mathbf{E})$.

Let $\left(\tau, X_{0}\right)$ be a random variable with law $P_{0}$ independent of a two-dimensional Brownian motion $\left(W_{t}\right)_{t \in[0, T]}$. According to $[\mathbf{1 6}]$, existence and trajectorial uniqueness hold for the stochastic differential equation with normal reflection

$$
\begin{gathered}
X_{t} \in \bar{\Theta}, \quad \forall t \in[0, T] \\
X_{t}=X_{0}+\sqrt{2 \nu} \int_{0}^{t} \mathbf{1}_{\{\tau \leqslant s\}} \mathrm{d} W_{s}-k_{t}, \\
|k|_{t}=\int_{0}^{t} \mathbf{1}_{\left\{X_{s} \in \partial \Theta\right\}} \mathbf{1}_{\{\tau \leqslant s\}} \mathrm{d}|k|_{s}, \quad k_{t}=\int_{0}^{t} n\left(X_{s}\right) \mathrm{d}|k|_{s} .
\end{gathered}
$$

Moreover, $\forall t \in[0, T], X_{t}$ admits

$$
x \rightarrow \frac{1}{\left\|w_{0}\right\|_{1}+\nu\|g\|_{L^{1}([0, t] \times \partial \Theta)}}\left(\left|w_{0}\right| P_{t}^{\nu}(x)+\nu \int_{0}^{t} \int_{\partial \Theta}|g|(s, y) P_{t-s}^{\nu}(y, x) \sigma(d y) \mathrm{d} s\right)
$$

as a density with respect to the Lebesgue measure on $\bar{\Theta}$. Since $\left\|K w_{s}\right\|_{L^{\infty}}$ is square integrable we deduce by Girsanov's theorem that the stochastic differential equation $\left(E^{w}\right)$ admits a weak solution $P$ such that $\forall t \in[0, T]$, the measure $\tilde{P}_{t}$ has a density. Let $\tilde{p}$ denote a measurable version of the densities.

We set $t \in[0, T]$. Reasoning as in the proof of Lemma 3.3, we obtain that for $\psi \in \mathcal{C}^{1,2}([0, t] \times \bar{\Theta})$ such that $\forall(s, x) \in[0, t] \times \partial \Theta, \partial_{n} \psi(s, x)=0$,

$$
\begin{aligned}
\int_{\bar{\Theta}} \psi(t, x) \tilde{p}(t, x) \mathrm{d} x= & \int_{\bar{\Theta}} \psi(0, x) w_{0}(x) \mathrm{d} x+\nu \int_{0}^{t} \int_{\partial \Theta} \psi(s, x) g(s, x) \mathrm{d} \sigma(x) \mathrm{d} s \\
& +\int_{0}^{t} \int_{\bar{\Theta}}\left(\partial_{s} \psi(s, x)+K w_{s}(x) \cdot \nabla \psi(s, x)+\nu \Delta \psi(s, x)\right) \tilde{p}(s, x) \mathrm{d} x \mathrm{~d} s .
\end{aligned}
$$

Choosing $\psi(s, x)=P_{t-s}^{\nu} \varphi(x)$ as in the proof of Proposition 2.7 and remarking that because of (2.24) and the uniform-in-time bound $\left\|\tilde{p}_{t}\right\|_{L^{1}} \leqslant\left\|w_{0}\right\|_{1}+\nu\|g\|_{1}$ (a consequence of $(3.4))$,

$$
\begin{aligned}
\int_{0}^{t} \int_{\Theta^{2}}\left|\nabla_{x} P_{t-s}^{\nu}(x, y)\right||\varphi(y)|\left|\tilde{p}_{s}(x)\right|\left|K w_{s}(x)\right| \mathrm{d} x \mathrm{~d} y \mathrm{~d} s & \leqslant C \int_{0}^{t} \frac{\left\|K w_{s}\right\|_{L^{\infty}} \mathrm{d} s}{\sqrt{t-s}} \\
& \leqslant C\|K w\|_{L_{t}^{4}\left(L_{x}^{\infty}\right)}<+\infty
\end{aligned}
$$

we deduce by Fubini's theorem that

$$
\begin{aligned}
\mathrm{d} x \text { a.e., } \quad \tilde{p}_{t}(x)=P_{t}^{\nu} w_{0}(x)+\int_{0}^{t} \nabla P_{t-s}^{\nu} \cdot\left(\tilde{p}_{s} K w_{s}\right)(x) \mathrm{d} s & \\
& +\nu \int_{(0, t] \times \partial \Theta} P_{t-s}^{\nu}(y, x) g(s, y) \mathrm{d} \sigma(y) \mathrm{d} s .
\end{aligned}
$$


Now, using the mild Equation (2.23) satisfied by $w$ and (2.24), we obtain

$$
\exists C>0, \quad \forall t \in[0, T], \quad\left\|\tilde{p}_{t}-w_{t}\right\|_{L^{1}} \leqslant C \int_{0}^{t}\left\|\tilde{p}_{s}-w_{s}\right\|_{L^{1}} \frac{\left\|K w_{s}\right\|_{L^{\infty}}}{\sqrt{t-s}} \mathrm{~d} s .
$$

By iterating this bound, then using Hölder's inequality, we obtain

$$
\begin{aligned}
\left\|\tilde{p}_{t}-w_{t}\right\|_{L^{1}} & \leqslant C \int_{0}^{t}\left\|\tilde{p}_{s}-w_{s}\right\|_{L^{1}}\left\|K w_{s}\right\|_{L^{\infty}} \int_{s}^{t} \frac{\left\|K w_{u}\right\|_{L^{\infty}} \mathrm{d} u}{\sqrt{t-u} \sqrt{u-s}} \mathrm{~d} s \\
& \leqslant C \int_{0}^{t}\left\|\tilde{p}_{s}-w_{s}\right\|_{L^{1}}\left\|K w_{s}\right\|_{L^{\infty}}\|K w\|_{L_{t}^{4}\left(L_{x}^{\infty}\right)}\left(\int_{s}^{t}((t-u)(u-s))^{-2 / 3} \mathrm{~d} u\right)^{3 / 4} \mathrm{~d} s .
\end{aligned}
$$

Hence (3.12) holds with $(t-s)^{-1 / 2}$ replaced by $(t-s)^{-1 / 4}$ in the right-hand side. After the next iteration we obtain that (3.12) holds with $(t-s)^{-1 / 2}$ replaced by 1 and conclude by Gronwall's lemma that $\forall t \in[0, T], \tilde{p}_{t}=w_{t}$.

\section{Stochastic approximations of the solution of the vortex equation}

\subsection{The case of a cut-off kernel}

As in Méléard [20], we introduce a cut-off kernel $K_{\varepsilon}$ preserving the properties (2.4). More precisely we consider a non-decreasing $\mathcal{C}^{2}$-function $\eta$ from $\mathbb{R}_{+}$to $\mathbb{R}_{+}$, such that $\eta(x)=x$ for $x \leqslant \frac{1}{2}$ and $\eta(x)=1$ for $x \geqslant 1$. For $0<\varepsilon \leqslant 1$, we set

$$
G_{\varepsilon}(x, y)=\eta\left(\frac{|x-y|^{3}}{\varepsilon^{3}}\right) G(x, y)
$$

and

$$
\begin{aligned}
K_{\varepsilon}(x, y) & =\nabla_{x}^{\perp} G_{\varepsilon}(x, y) \\
& =\eta\left(\frac{|x-y|^{3}}{\varepsilon^{3}}\right) K(x, y)+\eta^{\prime}\left(\frac{|x-y|^{3}}{\varepsilon^{3}}\right) \frac{3(x-y)^{\perp}|x-y|}{\varepsilon^{3}} G(x, y) .
\end{aligned}
$$

The following lemma states useful properties of this cut-off kernel.

Lemma 4.1.

$$
\left.\begin{array}{c}
\nabla_{x} \cdot K_{\varepsilon}(x, y)=0, \quad K_{\varepsilon}(x, y) \cdot n(x)=0 \quad \text { for } x \in \partial \Theta, \\
K_{\varepsilon}(x, y)=K(x, y) \quad \text { if }|x-y| \geqslant \varepsilon, \\
\forall x, y \in \bar{\Theta}, \quad\left|K_{\varepsilon}(x, y)\right| \leqslant \frac{C(1+|\ln | x-y||)}{|x-y|},
\end{array}\right\}
$$

where $C$ does not depend on $\varepsilon$.

(2) $\sup _{x \in \bar{\Theta}}\left\|K(x, \cdot)-K_{\varepsilon}(x, \cdot)\right\|_{L_{y}^{p}}$ tends to 0 as $\varepsilon$ tends to 0 as soon as $p<2$.

(3) For $\varepsilon$ sufficiently small, the kernel $K_{\varepsilon}$ is bounded by $M_{\varepsilon} \leqslant C|\ln \varepsilon| / \varepsilon$ and Lipschitz continuous in both variables with constant $L_{\varepsilon} \leqslant C|\ln \varepsilon| / \varepsilon^{2}$, where $C$ does not depend on $\varepsilon$. 
Proof. The two first properties in (1) are obvious and (2) is an easy consequence of (4.3).

By the estimate of $K$ given in Lemma 2.1 and the above definition of $\eta$, the norm of the first term on the right-hand side of (4.2) is smaller than

$$
C_{0}\left(\frac{1}{|x-y|} \wedge \sup _{r \in\left[0, \varepsilon 2^{-1 / 3}\right]} \frac{r^{2}}{\epsilon^{3}}\right) \leqslant C_{0}\left(\frac{1}{|x-y|} \wedge \frac{1}{\epsilon}\right) .
$$

By the estimate of $G$ in Lemma 2.1 and since $\eta^{\prime}(x)=0$ for $x>1$, the second term on the right-hand side of (4.2) is smaller than $3 C_{0}\left\|\eta^{\prime}\right\|_{\infty}$ times

$$
\left(\frac{1+|\ln | x-y||}{|x-y|} \wedge \sup _{r \in[0, \varepsilon]} \frac{r^{2}(1+|\ln (r)|)}{\varepsilon^{3}}\right) \leqslant\left(\frac{1+|\ln | x-y||}{|x-y|} \wedge \frac{1+|\ln (\varepsilon)|}{\varepsilon}\right)
$$

as $\varepsilon \leqslant 1$. We deduce both (4.3) and the upper bound in $C|\ln (\varepsilon)| / \varepsilon$. To prove that $K_{\varepsilon}$ is Lipschitz continuous, we use in a similar way Lemma 2.1 combined with the definition of $\eta$ to check that the gradient of each coordinate of $K_{\varepsilon}$ with respect to either $x$ or $y$ is bounded by $C|\ln (\varepsilon)| / \varepsilon^{2}$ (the contribution of the first term on the right-hand side of (4.2) is $C / \varepsilon^{2}$, whereas the contribution of the second term is $\left.C|\ln (\varepsilon)| / \varepsilon^{2}\right)$.

With a slight adaptation of [23] to take into account the random births on the boundary, we obtain the existence and pathwise uniqueness of the following interacting particle systems.

Definition 4.2. Consider a sequence $\left(B^{i}\right)_{i \in \mathbb{N}}$ of independent Brownian motions on $\mathbb{R}^{2}$ and a sequence of independent variables $\left(\tau^{i}, Z_{0}^{i}\right)_{i \in \mathbb{N}}$ with values in $[0, T] \times \bar{\Theta}$ distributed according to $P_{0}$, and independent of the Brownian motions. For a fixed $\varepsilon>0$, for each $n \in \mathbb{N}^{*}$, let us consider the interacting processes defined for $1 \leqslant i \leqslant n$ by

$$
\left.\begin{array}{c}
Z_{t}^{i n, \varepsilon} \in \bar{\Theta}, \quad \forall t \in[0, T], \\
Z_{t}^{i n, \varepsilon}=Z_{0}^{i}+\sqrt{2 \nu} \int_{0}^{t} \mathbf{1}_{\left\{\tau^{i} \leqslant s\right\}} \mathrm{d} B_{s}^{i}+\int_{0}^{t} \mathbf{1}_{\left\{\tau^{i} \leqslant s\right\}} K_{\varepsilon} \tilde{\mu}_{s}^{n, \varepsilon}\left(Z_{s}^{i n, \varepsilon}\right) \mathrm{d} s-k_{t}^{i n, \varepsilon}, \\
\left|k^{i n, \varepsilon}\right|_{t}=\int_{0}^{t} \mathbf{1}_{\left\{Z_{s}^{i n, \varepsilon} \in \partial \Theta\right\}} \mathbf{1}_{\left\{\tau^{i} \leqslant s\right\}} \mathrm{d}\left|k^{i n, \varepsilon}\right|_{s}, \quad k_{t}^{i n, \varepsilon}=\int_{0}^{t} n\left(Z_{s}^{i n, \varepsilon}\right) \mathrm{d}\left|k^{i n, \varepsilon}\right|_{s},
\end{array}\right\}
$$

where

$$
\tilde{\mu}_{s}^{n, \varepsilon}=\frac{1}{n} \sum_{j=1}^{n} h\left(\tau^{j}, Z_{0}^{j}\right) \mathbf{1}_{\left\{\tau^{j} \leqslant s\right\}} \delta_{Z_{s}^{j n, \varepsilon}}
$$

is the weighted empirical measure of the system at time $s$ and

$$
K_{\varepsilon} \tilde{\mu}_{s}^{n, \varepsilon}(z)=\frac{1}{n} \sum_{j=1}^{n} h\left(\tau^{j}, Z_{0}^{j}\right) \mathbf{1}_{\left\{\tau^{j} \leqslant s\right\}} K_{\varepsilon}\left(z, Z_{s}^{j n, \varepsilon}\right) .
$$

Let us remark that the particles either are born at time 0 inside the domain and evolve as diffusive particles with normal reflecting boundary conditions, or are born at a random 
time on the boundary of the domain and then evolve in the same way as particles born inside the domain. Moreover, all particles, as soon as they are born, interact together following a mean field depending on the parameter $\varepsilon$.

Again according to $[\mathbf{2 3}]$, we also get the existence and pathwise uniqueness of the limiting processes (when $n$ tends to infinity and $\varepsilon$ is fixed), coupled with the interacting processes, as follows.

Definition 4.3. For $i \geqslant 1$, we define $\bar{Z}^{i, \varepsilon}$ by

$$
\left.\begin{array}{c}
\bar{Z}_{t}^{i, \varepsilon} \in \bar{\Theta}, \quad \forall t \in[0, T], \\
\bar{Z}_{t}^{i, \varepsilon}=Z_{0}^{i}+\sqrt{2 \nu} \int_{0}^{t} \mathbf{1}_{\left\{\tau^{i} \leqslant s\right\}} \mathrm{d} B_{s}^{i}+\int_{0}^{t} \mathbf{1}_{\left\{\tau^{i} \leqslant s\right\}} K_{\varepsilon} \tilde{Q}_{s}^{\varepsilon}\left(\bar{Z}_{s}^{i, \varepsilon}\right) \mathrm{d} s-\bar{k}_{t}^{i, \varepsilon}, \\
\left|\bar{k}^{i, \varepsilon}\right|_{t}=\int_{0}^{t} \mathbf{1}_{\left\{\bar{Z}_{s}^{i, \varepsilon} \in \partial \Theta\right\}} \mathbf{1}_{\left\{\tau^{i} \leqslant s\right\}} \mathrm{d}\left|\bar{k}^{i, \varepsilon}\right|_{s}, \quad \bar{k}_{t}^{i, \varepsilon}=\int_{0}^{t} n\left(\bar{Z}_{s}^{i, \varepsilon}\right) \mathrm{d}\left|\bar{k}^{i, \varepsilon}\right|_{s},
\end{array}\right\}
$$

where for $(s, z) \in[0, T] \times \bar{\Theta}$,

$$
K_{\varepsilon} \tilde{Q}_{s}^{\varepsilon}(z)=\int_{\bar{\Theta}} K_{\varepsilon}(z, x) \tilde{Q}_{s}^{\varepsilon}(\mathrm{d} x)
$$

with the signed measure $\tilde{Q}_{s}^{\varepsilon}$ on $\bar{\Theta}$ defined from the common law $Q^{\varepsilon}$ of $\left(\tau^{i}, \bar{Z}^{i, \varepsilon}, \bar{k}^{i, \varepsilon}\right)$ by (3.4).

Sznitman also proves a propagation-of-chaos result, but without precise estimates on the rate of convergence. We need such estimates to prove our main result.

Proposition 4.4. For $t \leqslant T$, and for each $i \in\{1, \ldots, n\}$,

$$
\begin{aligned}
& E\left(\sup _{s \leqslant t}\left|Z_{s}^{i n, \varepsilon}-\bar{Z}_{s}^{i, \varepsilon}\right|^{2}\right) \leqslant 2 d(\Theta) \sqrt{\frac{A_{\varepsilon}}{n}} \exp \left(C_{H}\left(1+\left(\left\|w_{0}\right\|_{1}+\nu\|g\|_{1}\right)\left(M_{\varepsilon} / 2+L_{\varepsilon}\right) t\right)\right) \\
& E\left(\sup _{s \leqslant t}\left|k_{s}^{i n, \varepsilon}-\bar{k}_{s}^{i, \varepsilon}\right|\right) \leqslant E\left(\sup _{s \leqslant t}\left|Z_{s}^{i n, \varepsilon}-\bar{Z}_{s}^{i, \varepsilon}\right|\right) \\
& +2\left(\left\|w_{0}\right\|_{1}+\nu\|g\|_{1}\right) t\left(L_{\varepsilon} E\left(\sup _{s \leqslant t}\left|Z_{s}^{i n, \varepsilon}-\bar{Z}_{s}^{i, \varepsilon}\right|\right)+\frac{M_{\varepsilon}}{\sqrt{n}}\right),
\end{aligned}
$$

where $C_{H}$ is a constant which depends only on upper bounds of the function $H$ and its derivatives and

$$
A_{\varepsilon}=\frac{4\left(\left\|w_{0}\right\|_{1}+\nu\|g\|_{1}\right)^{2} M_{\varepsilon}^{2}}{2+\left(\left\|w_{0}\right\|_{1}+\nu\|g\|_{1}\right)\left(M_{\varepsilon}+2 L_{\varepsilon}\right)} .
$$

Remark 4.5. The convergence rate in the number $n$ of particles given above is not optimal: indeed one can check that $E\left(\sup _{s \leqslant t}\left|Z_{s}^{i n, \varepsilon}-\bar{Z}_{s}^{i, \varepsilon}\right|^{4}\right)$ is smaller than

$$
\begin{aligned}
& \frac{16\left(\left\|w_{0}\right\|_{1}+\|\nu g\|_{1}\right)^{4} M_{\varepsilon}^{4} t}{n^{2}\left(1+\left(2+\left(\left\|w_{0}\right\|_{1}+\nu\|g\|_{1}\right)^{2}\left(M_{\varepsilon}^{2}+4 L_{\varepsilon}^{2}\right)\right) t\right)} \\
& \times \exp \left(C_{H}\left(t+\left(2+\left(\left\|w_{0}\right\|_{1}+\nu\|g\|_{1}\right)^{2}\left(M_{\varepsilon}^{2}+4 L_{\varepsilon}^{2}\right)\right) t^{2}\right)\right) .
\end{aligned}
$$

But in the next section we are going to let $\varepsilon=\varepsilon_{n}$ depend on $n$ and converge to 0 in such a way that $E\left(\sup _{s \leqslant t}\left|Z_{s}^{i n, \varepsilon_{n}}-\bar{Z}_{s}^{i, \varepsilon_{n}}\right|^{2}\right) \rightarrow 0$. The estimate given in the proposition allows a quicker (but still very slow) convergence of $\varepsilon_{n}$ to 0 than the previous one. 
Proof. Let us compare the two processes $Z^{i n, \varepsilon}$ and $\bar{Z}^{i, \varepsilon}$. Integrating (3.6) over time with $Z=Z^{i n, \varepsilon}, k=k^{i n, \varepsilon}, \bar{Z}=\bar{Z}^{i, \varepsilon}$ and $\bar{k}=\bar{k}^{i, \varepsilon}, b_{t}=K_{\varepsilon} \tilde{\mu}_{t}^{n, \varepsilon}\left(Z_{t}\right)$ and $\bar{b}_{t}=K_{\varepsilon} \tilde{Q}_{t}^{\varepsilon}\left(\bar{Z}_{t}\right)$, and taking expectations, we obtain

$$
\begin{aligned}
E\left(\left|Z_{t}^{i n, \varepsilon}-\bar{Z}_{t}^{i, \varepsilon}\right|^{2}\right) \leqslant C_{H}( & \left(1+M_{\varepsilon}\left(\left\|w_{0}\right\|_{1}+\nu\|g\|_{1}\right)\right) \int_{0}^{t} E\left(\left|Z_{s}^{i n, \varepsilon}-\bar{Z}_{s}^{i, \varepsilon}\right|^{2}\right) \mathrm{d} s \\
& \left.+\int_{0}^{t} E\left(\left|Z_{s}^{i n, \varepsilon}-\bar{Z}_{s}^{i, \varepsilon}\right|\left|K_{\varepsilon} \tilde{\mu}_{s}^{n, \varepsilon}\left(Z_{s}^{i n, \varepsilon}\right)-K_{\varepsilon} \tilde{Q}_{s}^{\varepsilon}\left(\bar{Z}_{s}^{i, \varepsilon}\right)\right|\right) \mathrm{d} s\right) .
\end{aligned}
$$

Using the Lipschitz continuity of $K_{\varepsilon}$, the boundedness of $h$ and the exchangeability of the processes $\left(Z^{i n, \varepsilon}, \bar{Z}^{i, \varepsilon}\right), 1 \leqslant i \leqslant n$, we obtain

$$
\begin{aligned}
E\left(\left|Z_{s}^{i n, \varepsilon}-\bar{Z}_{s}^{i, \varepsilon}\right|\left|K_{\varepsilon} \tilde{\mu}_{s}^{n, \varepsilon}\left(Z_{s}^{i n, \varepsilon}\right)-K_{\varepsilon} \tilde{Q}_{s}^{\varepsilon}\left(\bar{Z}_{s}^{i, \varepsilon}\right)\right|\right) & \\
\leqslant & \left(\left\|w_{0}\right\|_{1}+\nu\|g\|_{1}\right) L_{\varepsilon} E\left(\left|Z_{s}^{i n, \varepsilon}-\bar{Z}_{s}^{i, \varepsilon}\right|\left(\left|Z_{s}^{i n, \varepsilon}-\bar{Z}_{s}^{i, \varepsilon}\right|+\frac{1}{n} \sum_{j=1}^{n}\left|Z_{s}^{j n, \varepsilon}-\bar{Z}_{s}^{j, \varepsilon}\right|\right)\right) \\
& +E\left(\left|Z_{s}^{i n, \varepsilon}-\bar{Z}_{s}^{i, \varepsilon}\right|\left|\frac{1}{n} \sum_{j=1}^{n} h\left(\tau_{j}, Z_{0}^{j}\right) \mathbf{1}_{\left\{\tau^{j} \leqslant s\right\}} K_{\varepsilon}\left(\bar{Z}_{s}^{i, \varepsilon}, \bar{Z}_{s}^{j, \varepsilon}\right)-K_{\varepsilon} \tilde{Q}_{s}^{\varepsilon}\left(\bar{Z}_{s}^{i, \varepsilon}\right)\right|\right) \\
\leqslant & \left(1+2\left(\left\|w_{0}\right\|_{1}+\nu\|g\|_{1}\right) L_{\varepsilon}\right) E\left(\left|Z_{s}^{i n, \varepsilon}-\bar{Z}_{s}^{i, \varepsilon}\right|^{2}\right) \\
& +E\left(\left|\frac{1}{n} \sum_{j=1}^{n} h\left(\tau_{j}, Z_{0}^{j}\right) \mathbf{1}_{\left\{\tau^{j} \leqslant s\right\}} K_{\varepsilon}\left(\bar{Z}_{s}^{i, \varepsilon}, \bar{Z}_{s}^{j, \varepsilon}\right)-K_{\varepsilon} \tilde{Q}_{s}^{\varepsilon}\left(\bar{Z}_{s}^{i, \varepsilon}\right)\right|^{2}\right) .
\end{aligned}
$$

After expansion of

$$
E\left(\left|\frac{1}{n} \sum_{j=1}^{n} h\left(\tau_{j}, Z_{0}^{j}\right) \mathbf{1}_{\left\{\tau^{j} \leqslant s\right\}} K_{\varepsilon}\left(\bar{Z}_{s}^{i, \varepsilon}, \bar{Z}_{s}^{j, \varepsilon}\right)-K_{\varepsilon} \tilde{Q}_{s}^{\varepsilon}\left(\bar{Z}_{s}^{i, \varepsilon}\right)\right|^{2}\right),
$$

the expectation of the non-diagonal terms disappears because of the independence of the variables which are centred conditionally to $\bar{Z}^{i, \varepsilon}$. There only remain the $n$ bounded diagonal terms. We deduce that

$$
\begin{aligned}
E\left(\left|Z_{t}^{i n, \varepsilon}-\bar{Z}_{t}^{i, \varepsilon}\right|^{2}\right) \leqslant C_{H}\left(\left(2+\left(\left\|w_{0}\right\|_{1}+\nu\|g\|_{1}\right)\left(M_{\varepsilon}+2 L_{\varepsilon}\right)\right)\right. & \int_{0}^{t} E\left(\left|Z_{s}^{i n, \varepsilon}-\bar{Z}_{s}^{i, \varepsilon}\right|^{2}\right) \mathrm{d} s \\
& \left.+\frac{4\left(\left\|w_{0}\right\|_{1}+\nu\|g\|_{1}\right)^{2} M_{\varepsilon}^{2} t}{n}\right) .
\end{aligned}
$$

Using Gronwall's lemma, we obtain that both sides of (4.6) and (4.7) are smaller than

$$
f(t)=\frac{4\left(\left\|w_{0}\right\|_{1}+\|\nu g\|_{1}\right)^{2} M_{\varepsilon}^{2}}{n\left(2+\left(\left\|w_{0}\right\|_{1}+\nu\|g\|_{1}\right)\left(M_{\varepsilon}+2 L_{\varepsilon}\right)\right)} \exp \left(C_{H}\left(2+\left(\left\|w_{0}\right\|_{1}+\nu\|g\|_{1}\right)\left(M_{\varepsilon}+2 L_{\varepsilon}\right)\right) t\right) .
$$

Integrating (3.6) with respect to time, dealing with the stochastic integral thanks to Doob's inequality and using the fact that the right-hand side of (4.6) is smaller than $f(t)$, 
we get

$$
\begin{aligned}
E\left(\sup _{s \leqslant t}\left|Z_{s}^{i n, \varepsilon}-\bar{Z}_{s}^{i, \varepsilon}\right|^{2}\right) \leqslant & \left(C_{H} \int_{0}^{t} E\left(\left|Z_{s}^{i n, \varepsilon}-\bar{Z}_{s}^{i, \varepsilon}\right|^{4}\right) \mathrm{d} s\right)^{1 / 2}+f(t) \\
\leqslant & d(\Theta)\left(C_{H} \int_{0}^{t} E\left(\left|Z_{s}^{i n, \varepsilon}-\bar{Z}_{s}^{i, \varepsilon}\right|^{2}\right) \mathrm{d} s\right)^{1 / 2}+f(t) \\
& \leqslant d(\Theta) \sqrt{f(t)}+f(t) \\
& \text { since the right-hand side of }(4.7) \text { is smaller than } f(t) .
\end{aligned}
$$

The left-hand side being smaller than $d(\Theta)^{2}$, it is smaller than $2 d(\Theta) \sqrt{f(t)}$ when $f(t) \geqslant$ $d(\Theta)^{2}$ and the right-hand side is smaller than $2 d(\Theta) \sqrt{f(t)}$ otherwise. We deduce the desired estimate for $E\left(\sup _{s \leqslant t}\left|Z_{s}^{i n, \varepsilon}-\bar{Z}_{s}^{i, \varepsilon}\right|^{2}\right)$.

Now remarking that

$$
\sup _{s \leqslant t}\left|k_{s}^{i n, \varepsilon}-\bar{k}_{s}^{i, \varepsilon}\right| \leqslant \int_{0}^{t}\left|K_{\varepsilon} \tilde{\mu}_{s}^{n, \varepsilon}\left(Z_{s}^{i n, \varepsilon}\right)-K_{\varepsilon} \tilde{Q}_{s}^{\varepsilon}\left(\bar{Z}_{s}^{i, \varepsilon}\right)\right| \mathrm{d} s+\sup _{s \leqslant t}\left|Z_{s}^{i n, \varepsilon}-\bar{Z}_{s}^{i, \varepsilon}\right|
$$

and using arguments developed above we obtain the second estimate.

Remark 4.6. Let us remark that if $\bar{\Theta}$ is a convex region, then the rate of convergence is easier to obtain. Indeed the constant $C_{\mathrm{sp}}$ defined in (2.6) can be chosen equal to 0:

$$
\forall x \in \partial \Theta, \quad \forall x^{\prime} \in \bar{\Theta}, \quad n(x) \cdot\left(x-x^{\prime}\right) \geqslant 0 .
$$

In the expression $\left|Z_{t}^{i n, \varepsilon}-\bar{Z}_{t}^{i, \varepsilon}\right|^{2}$ given by Itô's formula, the local-times terms are nonpositive and therefore

$$
\begin{aligned}
\left.E\left(\sup _{s \leqslant t}\left|Z_{s}^{i n, \varepsilon}-\bar{Z}_{s}^{i, \varepsilon}\right|^{2}\right) \leqslant\left(1+2\left(\left\|w_{0}\right\|_{1}+\nu\|g\|_{1}\right) L_{\varepsilon}\right)\right) \int_{0}^{t} E\left(\sup _{u \leqslant s}\left|Z_{u}^{i n, \varepsilon}-\bar{Z}_{u}^{i, \varepsilon}\right|^{2}\right) \mathrm{d} s \\
+\frac{4\left(\left\|w_{0}\right\|_{1}+\nu\|g\|_{1}\right)^{2} M_{\varepsilon}^{2} t}{n}
\end{aligned}
$$

and we conclude by Gronwall's lemma.

\subsection{Convergence of the nonlinear processes}

For $i \geqslant 1$, let us denote by $\left(X^{i}, k^{i}\right)$ the solution of the nonlinear stochastic differential equation (E) with initial variable $\left(\tau^{i}, Z_{0}^{i}\right)$ and Brownian motion $B^{i}$. We now want to prove the pathwise convergence of $\left(\bar{Z}^{i, \varepsilon}, \bar{k}^{i, \varepsilon}\right)$ to $\left(X^{i}, k^{i}\right)$ when $\varepsilon$ tends to 0 .

By Girsanov's theorem, it turns out that $\forall t \in[0, T]$, the signed measure $\tilde{Q}_{t}^{\varepsilon}$ has a density $q_{t}^{\varepsilon}$ with respect to the Lebesgue measure on $\Theta$. Reasoning as in the proof of Theorem 3.4 and using the boundedness of the kernel $K_{\varepsilon}$, we show that $q^{\varepsilon}$ is the unique solution in $L_{T}^{1}=\left\{p_{t},\|p\|_{T}=\sup _{t \leqslant T}\left\|p_{t}\right\|_{L^{1}}<+\infty\right\}$ of the evolution equation

$$
q_{t}^{\varepsilon}(x)=P_{t}^{\nu} w_{0}(x)+\int_{0}^{t} \nabla_{x} P_{t-s}^{\nu} \cdot\left(q_{s}^{\varepsilon} K_{\varepsilon} q_{s}^{\varepsilon}\right)(x) \mathrm{d} s+\nu \int_{0}^{t} \int_{\partial \Theta} P_{t-s}^{\nu} g(s, y) \mathrm{d} \sigma(y) \mathrm{d} s .
$$


On the other hand, thanks to (4.3), we can apply all that we have done for Equation (2.1) to the equation

$$
\left.\begin{array}{c}
\partial_{t} w(t, x)+\nabla \cdot\left(w K_{\varepsilon} w\right)(t, x)=\nu \Delta w(t, x) \quad \text { in } \Theta, \\
w(x, 0)=w_{0} \quad \text { in } \Theta, \\
\partial_{n} w=\nabla w \cdot n=g \quad \text { on } \partial \Theta,
\end{array}\right\}
$$

We can then prove the existence of a unique weak solution $w^{\varepsilon}$ belonging to $L_{t}^{\infty}\left(L_{x}^{2}\right) \cap$ $L_{t}^{2}\left(H_{x}^{1}\right)$. Now, as in Proposition 2.7, we obtain that $w^{\varepsilon}$ is also a solution of (4.9). Since it belongs to $L_{T}^{1}$ ( $\Theta$ is bounded), $w^{\varepsilon}=q^{\varepsilon}$. Thanks to (4.3), one can check that the $a$ priori estimate $(2.20)$ holds for $w^{\varepsilon}=q^{\varepsilon}$ with a constant $C_{T}$ independent of $\varepsilon$. Following Remark 2.5, we deduce that

$$
\sup _{\varepsilon \in(0,1]}\left(\left\|q^{\varepsilon}\right\|_{L_{t}^{\infty}\left(L_{x}^{2}\right)}+\left\|q^{\varepsilon}\right\|_{L_{t}^{2}\left(H_{x}^{1}\right)}+\left\|\partial_{t} q^{\varepsilon}\right\|_{L_{t}^{2}\left(H_{x}^{1^{\prime}}\right)}+\left\|q^{\varepsilon}\right\|_{L_{t}^{4}\left(L_{x}^{4}\right)}\right)<+\infty .
$$

We can now prove the convergence of $q^{\varepsilon}$ to $w$.

Proposition 4.7. For each $1<p<+\infty$,

$$
\lim _{\varepsilon \rightarrow 0}\left\|q^{\varepsilon}-w\right\|_{L_{t}^{2}\left(L_{x}^{p}\right)}=0, \quad \lim _{\varepsilon \rightarrow 0}\left\|K_{\varepsilon} q^{\varepsilon}-K w\right\|_{L_{t}^{2}\left(L_{x}^{\infty}\right)}=0 .
$$

Proof. Let $\left(\varepsilon_{n}\right)_{n}$ be a sequence of positive numbers converging to 0 as $n$ tends to $+\infty$. Thanks to (4.11) and using the same compacity result as in the proof of Theorem 2.6, one can extract (by a diagonal procedure) from the sequence $\left(q^{\varepsilon_{n}}\right)_{n}$ a subsequence (still denoted $\left(q^{\varepsilon_{n}}\right)_{n}$ for simplicity), which converges strongly in $L_{t}^{2}\left(L_{x}^{p}\right)$, for each $p \in(1,+\infty)$, weakly in $L_{t}^{2}\left(H_{x}^{1}\right)$ and weakly* in $L_{t}^{\infty}\left(L_{x}^{2}\right)$ to $\tilde{w}$.

The strong convergence in $L_{t}^{2}\left(L_{x}^{p}\right)$ with $p>2$ combined with Lemma 4.1(2) and Hölder's inequality ensures that $K_{\varepsilon_{n}} q^{\varepsilon_{n}}$ converges to $K \tilde{w}$ in $L_{t}^{2}\left(L_{x}^{\infty}\right)$. By adapting the proof of Theorem 2.6, we get that $\tilde{w}$ is a weak solution of (2.1) and deduce that $\tilde{w}=w$ by uniqueness for this equation. We conclude since the sequence $\left(\varepsilon_{n}\right)_{n}$ is arbitrary.

We now study the convergence of the processes $\left(\bar{Z}^{i, \varepsilon_{n}}, \bar{k}^{i, \varepsilon_{n}}\right)$ as $n$ tends to $\infty$.

Theorem 4.8. For any $i \geqslant 1$, the processes $\left(\bar{Z}^{i, \varepsilon}, \bar{k}^{i, \varepsilon}\right)$ pathwisely converge to the solution $\left(X^{i}, k^{i}\right)$ of $(\boldsymbol{E})$ with initial variable $\left(\tau^{i}, Z_{0}^{i}\right)$ and Brownian motion $B^{i}$, as $\varepsilon$ tends to 0:

$$
\lim _{\varepsilon \rightarrow 0} E\left(\sup _{t \leqslant T}\left(\left|\bar{Z}_{t}^{i, \varepsilon}-X_{t}^{i}\right|^{2}+\left|\bar{k}_{t}^{i, \varepsilon}-k_{t}^{i}\right|\right)\right)=0 .
$$

Proof. For notational simplicity and since the law of $\left(\bar{Z}^{i, \varepsilon}, \bar{k}^{i, \varepsilon}, X^{i}, k^{i}\right)$ does not depend on $i$ we remove the superscript $i$ in the proof. Integrating (3.6) over time with $\bar{Z}=\bar{Z}^{\varepsilon}, Z_{t}=X, \bar{b}_{t}=K_{\varepsilon} q_{t}^{\varepsilon}\left(\bar{Z}_{t}^{\varepsilon}\right)$ and $b_{t}=K w_{t}\left(X_{t}\right)$, taking expectations and using (2.15), 
which also holds with $K_{\varepsilon}$ replacing $K$ (see Lemma 4.1(2)), we obtain

$$
\begin{aligned}
& E\left(\left|\bar{Z}_{t}^{\varepsilon}-X_{t}\right|^{2}\right) \\
& \leqslant C \int_{0}^{t}\left(1+\left\|K_{\varepsilon} q_{s}^{\varepsilon}\right\|_{L^{\infty}}+\left\|K w_{s}\right\|_{L^{\infty}}\right) E\left(\left|\bar{Z}_{s}^{\varepsilon}-X_{s}\right|^{2}\right) \mathrm{d} s \\
& \quad+C \int_{0}^{t} E\left(\left|\bar{Z}_{s}^{\varepsilon}-X_{s}\right|\left|K_{\varepsilon} q_{s}^{\varepsilon}\left(\bar{Z}_{s}^{\varepsilon}\right)-K w_{s}\left(X_{s}\right)\right|\right) \mathrm{d} s \\
& \leqslant C \int_{0}^{t}\left(1+\left\|q_{s}^{\varepsilon}\right\|_{H^{1}}+\left\|w_{s}\right\|_{H^{1}}\right) E\left(\left|\bar{Z}_{s}^{\varepsilon}-X_{s}\right|^{2}\right) \mathrm{d} s \\
& \quad+C \int_{0}^{t} E\left(\left|\bar{Z}_{s}^{\varepsilon}-X_{s}\right|\left(\left|K_{\varepsilon} q_{s}^{\varepsilon}\left(\bar{Z}_{s}^{\varepsilon}\right)-K w_{s}\left(\bar{Z}_{s}^{\varepsilon}\right)\right|+\left|K w_{s}\left(\bar{Z}_{s}^{\varepsilon}\right)-K w_{s}\left(X_{s}\right)\right|\right)\right) \mathrm{d} s .
\end{aligned}
$$

Reasoning exactly as in the derivation of (3.8), we get

$$
E\left(\left|\bar{Z}_{s}^{\varepsilon}-X_{s}\right|\left|K w_{s}\left(\bar{Z}_{s}^{\varepsilon}\right)-K w_{s}\left(X_{s}\right)\right|\right) \leqslant C\left\|w_{s}\right\|_{H^{1}} \phi\left(E\left(\frac{\left|\bar{Z}_{s}^{\varepsilon}-X_{s}\right|^{2}}{d(\Theta)^{2}}\right)\right) .
$$

Moreover,

$$
E\left(\left|\bar{Z}_{s}^{\varepsilon}-X_{s}\right|\left|K_{\varepsilon} q_{s}^{\varepsilon}\left(\bar{Z}_{s}^{\varepsilon}\right)-K w_{s}\left(\bar{Z}_{s}^{\varepsilon}\right)\right|\right) \leqslant d(\Theta)\left\|K_{\varepsilon} q_{s}^{\varepsilon}-K w_{s}\right\|_{L^{\infty}} .
$$

Defining $y_{\varepsilon}(s)=E\left(\left|\bar{Z}_{s}^{\varepsilon}-X_{s}\right|^{2}\right) / d(\Theta)^{2}$, we deduce that $\forall t \leqslant T$,

$$
y_{\varepsilon}(t) \leqslant \zeta_{\varepsilon}+C \int_{0}^{t}\left(1+\left\|q_{s}^{\varepsilon}\right\|_{H^{1}}+\left\|w_{s}\right\|_{H^{1}}\right)\left(y_{\varepsilon}(s)+\phi\left(y_{\varepsilon}(s)\right)\right) \mathrm{d} s,
$$

where

$$
\zeta_{\varepsilon}=C \int_{0}^{T}\left\|K_{\varepsilon} q_{s}^{\varepsilon}-K \tilde{w}_{s}\right\|_{L^{\infty}} \mathrm{d} s \rightarrow_{\varepsilon \rightarrow 0} 0,
$$

according to Proposition 4.7. Arguing as in the proof of Theorem 3.4, one obtains that $\forall t \in[0, T], y_{\varepsilon}(t) \leqslant z_{\varepsilon}(t)$, where $z_{\varepsilon}$ is a non-decreasing solution of the ordinary differential equation

$$
z_{\varepsilon}^{\prime}(t)=C \int_{0}^{t}\left(1+\left\|q_{s}^{\varepsilon}\right\|_{H^{1}}+\left\|w_{s}\right\|_{H^{1}}\right)\left(z_{\varepsilon}(s)+\phi\left(z_{\varepsilon}(s)\right)\right) \mathrm{d} s, \quad z_{\varepsilon}(0)=\zeta_{\varepsilon} .
$$

According to (4.11),

$$
\int_{\zeta_{\varepsilon}}^{z_{\varepsilon}(T)} \frac{\mathrm{d} u}{u+\phi(u)} \leqslant C \sup _{\varepsilon} \int_{0}^{T}\left(1+\left\|q_{s}^{\varepsilon}\right\|_{H^{1}}+\left\|w_{s}\right\|_{H^{1}}\right) \mathrm{d} s<+\infty .
$$

With (4.14) and since

$$
\int_{0^{+}} \frac{\mathrm{d} u}{u+\phi(u)}=+\infty
$$

we deduce that $z_{\varepsilon}(T)=\sup _{t \in[0, T]} z_{\varepsilon}(t) \rightarrow 0$ as $\varepsilon$ tends to zero and conclude that $\sup _{t \in[0, T]} y_{\varepsilon}(t) \rightarrow 0$. 
As $\phi$ is non-decreasing, the right-hand side of (4.13) is smaller than

$$
\zeta_{\varepsilon}+C \int_{0}^{t}\left(1+\left\|q_{s}^{\varepsilon}\right\|_{H^{1}}+\left\|w_{s}\right\|_{H^{1}}\right)\left(z_{\varepsilon}(s)+\phi\left(z_{\varepsilon}(s)\right)\right) \mathrm{d} s=z_{\varepsilon}(t) .
$$

Using this bound, we conclude the proof by arguments similar to the ones given at the end of the proof of Proposition 4.4.

\subsection{The convergence theorem}

We now consider a sequence $\left(\varepsilon_{n}\right)$ tending to 0 as $n$ tends to infinity, in such a way that

$$
\lim _{n \rightarrow+\infty} L_{\varepsilon_{n}}^{2} \sqrt{\frac{A_{\varepsilon_{n}}}{n}} \exp \left(C_{H}\left(1+\left(\left\|w_{0}\right\|_{1}+\nu\|g\|_{1}\right)\left(M_{\varepsilon_{n}} / 2+L_{\varepsilon_{n}}\right) T\right)\right)+\frac{M_{\varepsilon_{n}}^{2}}{n}=0 .
$$

This is possible, even if the convergence of $\varepsilon_{n}$ to 0 is then very slow. Let us now consider for each $n$ the system of processes $\left(\tau^{i}, Z^{i n}, k^{i n}\right)$, where $Z^{i n}=Z^{i n, \varepsilon_{n}}$ and $k^{i n}=k^{i n, \varepsilon_{n}}$ are defined in (4.4). The solutions $\left(X^{i}, k^{i}\right)_{1 \leqslant i}$ of $(\mathbf{E})$ with initial variable $\left(\tau^{i}, Z_{0}^{i}\right)$ and Brownian motion $B^{i}$ are defined on the same probability space. We can then state our main theorem.

\section{Theorem 4.9.}

(1) For each $i \geqslant 1$, one has

$$
\lim _{n \rightarrow+\infty} E\left(\sup _{t \in[0, T]}\left|Z_{t}^{i n}-X_{t}^{i}\right|^{2}+\sup _{t \in[0, T]}\left|k_{t}^{i n}-k_{t}^{i}\right|\right)=0 .
$$

In particular, the laws of the $n$-particle system $\left(\tau^{i}, Z^{\text {in }}, k^{i n}\right)_{1 \leqslant i \leqslant n}$, are P-chaotic, where $P$ denotes the law of the solution of the nonlinear equation $(\boldsymbol{E})$.

(2) The approximate velocity field converges to $K w$ :

$$
\lim _{n \rightarrow+\infty} E\left(\left\|K_{\varepsilon_{n}} \tilde{\mu}_{t}^{n, \varepsilon_{n}}(x)-K w_{t}(x)\right\|_{L_{t}^{2}\left(L_{x}^{\infty}\right)}^{2}\right)=0 .
$$

Proof. (1) This assertion is immediate, if we combine Proposition 4.4 and Theorem 4.8 .

(2) On the other hand,

$$
\begin{gathered}
E\left(\left|K_{\varepsilon_{n}} \tilde{\mu}_{t}^{n, \varepsilon_{n}}(x)-K w_{t}(x)\right|^{2}\right) \\
\leqslant 3 E\left(\left|K_{\varepsilon_{n}} \tilde{\mu}_{t}^{n, \varepsilon_{n}}(x)-\frac{1}{n} \sum_{i=1}^{n} \mathbf{1}_{\left\{\tau^{i} \leqslant t\right\}} h\left(\tau^{i}, Z_{0}^{i}\right) K_{\varepsilon_{n}}\left(x, \bar{Z}_{t}^{i, \varepsilon_{n}}\right)\right|^{2}\right. \\
+\left|\frac{1}{n} \sum_{i=1}^{n} \mathbf{1}_{\left\{\tau^{i} \leqslant t\right\}} h\left(\tau^{i}, Z_{0}^{i}\right) K_{\varepsilon_{n}}\left(x, \bar{Z}_{t}^{i, \varepsilon_{n}}\right)-K_{\varepsilon_{n}} \tilde{Q}_{t}^{\varepsilon_{n}}(x)\right|^{2} \\
\left.+\left|K_{\varepsilon_{n}} \tilde{Q}_{t}^{\varepsilon_{n}}(x)-K w_{t}(x)\right|^{2}\right) \\
\leqslant 3\left(\left(\left\|w_{0}\right\|_{1}+\nu\|g\|_{1}\right)^{2}\left(L_{\varepsilon_{n}}^{2} E\left(\sup _{s \leqslant t}\left|Z_{s}^{i n}-\bar{Z}_{s}^{i, \varepsilon_{n}}\right|^{2}\right)+\frac{4 M_{\varepsilon_{n}}^{2}}{n}\right)\right. \\
\left.+\left|K_{\varepsilon_{n}} q_{t}^{\varepsilon_{n}}(x)-K w_{t}(x)\right|^{2}\right) .
\end{gathered}
$$

We conclude using (4.15), Proposition 4.4 and Proposition 4.7. 
Remark 4.10. The propagation-of-chaos result is equivalent to the convergence in probability of the empirical measures to $P$, considered as probability measures on the path space (cf. [24]). As a consequence, if the space of finite measures on $\bar{\Theta}$ is endowed with the weak convergence topology, then for $t \in[0, T]$, the random finite measures

$$
\tilde{\mu}_{t}^{n, \varepsilon_{n}}=\frac{1}{n} \sum_{i=1}^{n} \mathbf{1}_{\left\{\tau^{i} \leqslant t\right\}} h\left(\tau^{i}, Z_{0}^{i}\right) \delta_{Z_{t}^{i n}}
$$

converge in probability to $w_{t}(x) \mathrm{d} x, w$ being the unique solution of the vortex equation.

\section{A numerical example}

Let us first exhibit an explicit solution of (2.1) on the unit disk $\Theta=B(0,1)$. For this, we use the Bessel function

$$
J_{0}(r)=\sum_{j \geqslant 0} \frac{(-1)^{j}}{(j !)^{2}}\left(\frac{1}{2} r\right)^{2 j} \quad \text { which solves } \frac{1}{r} \frac{\mathrm{d}}{\mathrm{d} r} r \frac{\mathrm{d}}{\mathrm{d} r} J_{0}(r)=-J_{0}(r) .
$$

We work with polar coordinates and set

$$
w(t, r, \theta)=\mathrm{e}^{-\nu t} J_{0}(r), \quad w_{0}(r, \theta)=J_{0}(r) \quad \text { and } \quad g(t, \theta)=\mathrm{e}^{-\nu t} J_{0}^{\prime}(1) .
$$

By (5.1),

$$
\Delta w(t, r, \theta)=\left(\frac{1}{r} \frac{\partial}{\partial r} r \frac{\partial}{\partial r}+\frac{1}{r^{2}} \frac{\partial^{2}}{\partial \theta^{2}}\right) w(t, r, \theta)=-w(t, r, \theta) .
$$

Hence the function $w(t, r, \theta)$ solves

$$
\left.\left.\partial_{t} w=\nu \Delta w \quad \text { in }\right] 0, T\right] \times B(0,1), \quad w(0, \cdot)=w_{0}(\cdot) \quad \text { in } B(0,1)
$$

and

$$
\left.\left.\partial_{n} w=g \quad \text { on }\right] 0, T\right] \times S(0,1) .
$$

In addition, by (5.1), $G w(t, r, \theta)=\mathrm{e}^{-\nu t}\left(J_{0}(1)-J_{0}(r)\right)$. Therefore,

$$
K w(t, r, \theta)=\nabla^{\perp} G w(t, r, \theta)=-\mathrm{e}^{-\nu t} J_{0}^{\prime}(r)(-\cos (\theta), \sin (\theta))
$$

is orthoradial and $K w \cdot \nabla w=0$ in $] 0, T] \times B(0,1)$, which implies that $w$ solves (2.1).

We choose $T=1$ and $\nu=\ln (2)$ so that the final vorticity and velocity fields are half the initial ones and discretize the dynamics of the particle system with time step $\Delta t=T / L$ with $L=100$. Since $J_{0}$ is positive on $[0,1]$ and $J_{0}^{\prime}(1)$ is negative, using $(2.1)$ one obtains

$$
\begin{gathered}
\left\|w_{0}\right\|_{1}=2 \pi \int_{0}^{1} r J_{0}(r) \mathrm{d} r=-2 \pi \int_{0}^{1} \frac{\mathrm{d}}{\mathrm{d} r} r \frac{\mathrm{d}}{\mathrm{d} r} J_{0}(r) \mathrm{d} r=-2 \pi J_{0}^{\prime}(1), \\
\|g\|_{1}=-2 \pi J_{0}^{\prime}(1) \int_{0}^{T} \mathrm{e}^{-\nu s} \mathrm{~d} s=-\frac{\pi}{\nu} J_{0}^{\prime}(1) \quad \text { and } \quad\left\|w_{0}\right\|_{1}+\nu\|g\|_{1}=3 \pi\left|J_{0}^{\prime}(1)\right| .
\end{gathered}
$$

We choose $n=30000$ particles. 

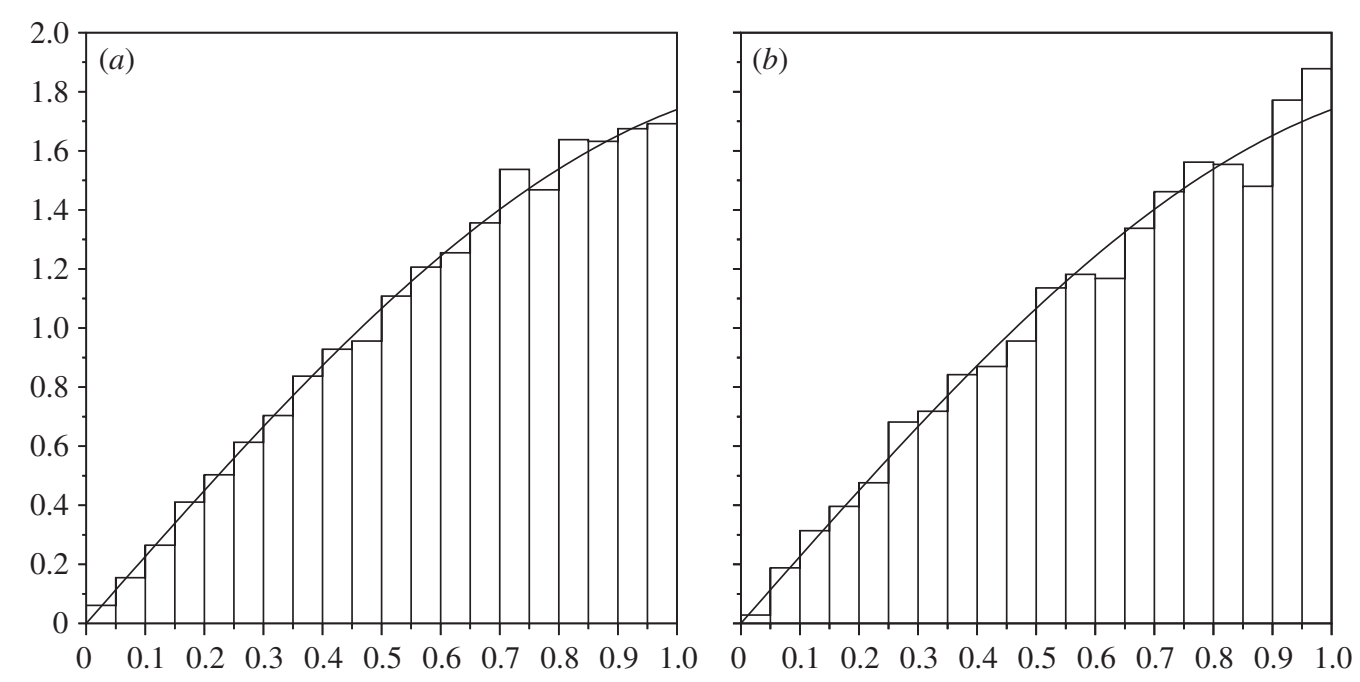

Figure 1. (a) Initial and (b) final repartition of norms of positions (exact, solid line; approximate, histogram).

- At time 0 , the positions $\left(Z_{0}^{i}\right)_{0 \leqslant i \leqslant N_{0}}$ of $N_{0}=n *\left(\left\|w_{0}\right\|_{1} /\left(\left\|w_{0}\right\|_{1}+\nu\|g\|_{1}\right)\right)=$ 20000 particles with positive weight $3 \pi\left|J_{0}^{\prime}(1)\right|$ are generated independently according to the density $1_{B(0,1)}(z) J_{0}(|z|) /\left(2 \pi\left|J_{0}^{\prime}(1)\right|\right)$.

- Inductively, for $0 \leqslant l \leqslant L-1$, the positions $\left(Z_{l \Delta t}^{i}\right)_{1 \leqslant i \leqslant N_{l}}$ of the $N_{l}$ particles alive at time $l \Delta t$ are updated according to the version of the Euler scheme with weak order of convergence $\Delta t$ proposed by Gobet [12]. More precisely, for $1 \leqslant i \leqslant N_{l}$,

- the velocity

$$
V_{l}^{i}=\frac{3 \pi\left|J_{0}^{\prime}(1)\right|}{n} \sum_{\substack{j=1 \\ j \neq i}}^{N_{l}}\left(1_{\left\{j \leqslant N_{0}\right\}}-1_{\left\{j>N_{0}\right\}}\right) K\left(Z_{l \Delta t}^{i}, Z_{l \Delta t}^{j}\right)
$$

is computed without cutting off $K$ but by removing the contribution of particle $i$ itself;

- if $\left|Z_{l \Delta t}^{i}\right| \leqslant 0.7$, we set $Z_{(l+1) \Delta t}^{i}=z_{l+1}^{i} * \min \left(1,1 /\left|z_{l+1}^{i}\right|\right)$, where

$$
z_{l+1}^{i}=Z_{l \Delta t}^{i}+\sqrt{2 \nu \Delta t} G_{l+1}^{i}+V_{l}^{i} \Delta t,
$$

the variables $\left(G_{l}^{i}\right)_{i \leqslant n, l \leqslant L}$ being i.i.d. according to the normal law on $\mathbb{R}^{2}$ - the choice of the parameter 0.7 ensures that the event $\left|z_{l+1}^{i}\right| \geqslant 1$ is very rare;

- if $\left|Z_{l \Delta t}^{i}\right|>0.7$, then a term corresponding to the exact normal reflection of the diffusion with constant diffusion coefficient $\sqrt{2 \nu}$ and drift coefficient $V_{l}^{i}$ on the line tangent to the unit circle at $Z_{l \Delta t}^{i} /\left|Z_{l \Delta t}^{i}\right|$ is added in the above expression of $z_{l+1}^{i}$ (see [12] for details). 



Figure 2. (a) Initial and (b) final velocities (exact and approximate).

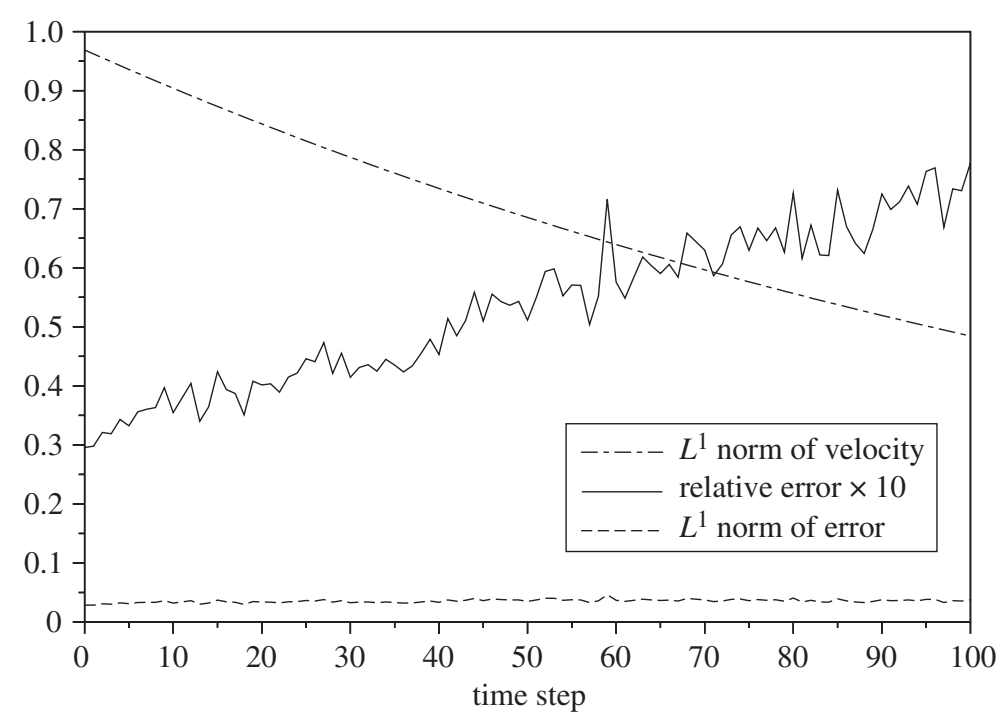

Figure 3. Evolution of error with respect to time step.

Then, at time $(l+1) \Delta t$, the positions $\left(Z_{(l+1) \Delta t}^{i}\right)_{N_{l}+1 \leqslant N_{l+1}}$ of $N_{l+1}-N_{l}=$ $\left[N_{0}\left(\mathrm{e}^{-\nu l \Delta t}-\mathrm{e}^{-\nu(l+1) \Delta t}\right)\right]$ particles with negative weight $3 \pi J_{0}^{\prime}(1)$ are independently generated uniformly on the unit circle.

Normalized histograms (the total area is equal to 1 ) in Figure 1 show the initial and final repartitions of norms of positions of particles. More precisely, since at the final time, positive and negative particles coexist, we count the numbers of positive and negative particles with norm in each interval and subtract the latter from the former. The 
histogram is drawn according to the result. The normalized exact density is given by $r \rightarrow r J_{0}(r) /\left|J_{0}^{\prime}(1)\right|$.

In Figure 2, the approximate velocity field computed from the particles is compared with the exact one given by (5.2) at both the initial and the final times. Lastly, Figure 3 gives the evolution with respect to time of the $L^{1}$ norm of exact velocity, of the $L^{1}$ norm of the velocity error, and of the relative error multiplied by 10. Of course, the $L^{1}$ norm of velocity decreases as $\mathrm{e}^{-\nu t}$. The $L^{1}$ norm of the error fluctuates around its initial value. And the relative error increases mainly because the $L^{1}$ norm of the velocity decreases.

Acknowledgements. We thank Tony Lelièvre for numerous discussions concerning the variational approach for evolution equations.

\section{References}

1. C. R. Anderson, Vorticity boundary conditions and boundary vorticity generation for two-dimensional viscous incompressible flows, J. Comput. Phys. 80 (1989), 72-97.

2. S. Benachour, B. Roynette and P. Vallois, Branching process associated with 2dNavier Stokes equation, Rev. Mat. Iberoamer. 17(2) (2001), 331-373.

3. G. Benfatto and M. Pulvirenti, Generation of vorticity near the boundary in planar Navier-Stokes flows, Commun. Math. Phys. 96 (1984), 59-95.

4. M. Bossy And B. Jourdain, Rate of convergence of a particle method for the solution of a 1d viscous scalar conservation law in a bounded interval, Ann. Probab. 30(4) (2002), 1797-1832.

5. H. Brezis, Analyse fonctionnelle (Masson, 1993).

6. A. J. Chorin, Numerical study of slightly viscous flows, J. Fluid Mech. 57 (1973), 785793.

7. G. H. Соттет, A vorticity creation algorithm, in Mathematical aspects of vortex dynamics (SIAM, Philadelphia, PA, 1989).

8. G. H. Соттет, Boundary conditions and deterministic vortex methods for the NavierStokes equations, in Navier-Stokes equations and related nonlinear problems (Plenum, New York, 1995).

9. B. Fernandez and S. MélÉard, Asymptotic behaviour for interacting diffusion processes with space-time random birth, Bernoulli 6(1) (2000), 91-111.

10. J. S. GIET, Processus stochastiques: application à l'équation de Navier-Stokes; simulation de la loi de diffusions irrégulières; vitesse du schéma d'Euler pour des fonctionnelles, Thèse de doctorat de l'Université Henri Poincaré, Nancy 1 (2000).

11. D. Gilbarg and N. S. Trudinger, Elliptic partial differential equations of second order, 2nd edn (Springer, 1983).

12. E. Goвet, Euler schemes and half-space approximations for the simulation of diffusion in a domain, ESAIM Probab. Stat. 5 (2001), 261-293.

13. B. Jourdain, Diffusion processes associated with nonlinear evolution equations for signed initial measures, Methodol. Comput. Appl. Prob. 2(1) (2000), 69-91.

14. O. A. Ladyzenskaja, V. A. Solonnikov and N. N. Ural'CeVa, Linear and quasilinear equations of parabolic type (American Mathematical Society, 1968).

15. P. L. Lions, Quelques méthodes de résolution des problèmes aux limites non linéaires (Dunod, 1969).

16. P. L. Lions And A. S. Sznitman, Stochastic differential equations with reflecting boundary conditions, Commun. Pure Appl. Math. 37 (1984), 511-537.

17. C. Marchioro and M. Pulvirenti, Hydrodynamics in two dimensions and vortex theory, Commun. Math. Phys. 84 (1982), 483-503. 
18. S. MÉLÉARD, Asymptotic behaviour of some interacting particle systems, McKean-Vlasov and Boltzmann models, CIME Lectures, Lecture Notes in Mathematics, no. 1627, pp. 4295 (Springer, 1996).

19. S. MÉLÉARD, A trajectorial proof of the vortex method for the two-dimensional NavierStokes equation, Ann. Appl. Probab. 10(4) (2000), 1197-1211.

20. S. MÉLÉARD, Monte Carlo approximations of the solution of two-dimensional NavierStokes equations with finite measure initial data, Probab. Theory Relat. Fields 121(3) (2001), 367-388.

21. Ch. Pommerenke, Boundary behaviour of conformal maps (Springer, 1992).

22. K. Sato And T. Ueno, Multi-dimensional diffusion and the Markov process on the boundary, J. Math. Kyoto Univ. 4(3) (1965), 529-605.

23. A. S. Sznitman, Nonlinear reflecting diffusion process, and the propagation of chaos and fluctuations associated, J. Funct. Analysis 56 (1984), 311-336.

24. A. S. Sznitman, Topics in propagation of chaos, Ecole d'été de Probabilités de SaintFlour XIX (1989), Lecture Notes in Mathematics, no. 1464 (Springer, 1991).

25. R. Temam, Navier-Stokes equations (North Holland, Amsterdam, 1979).

26. L. A. YING, Convergence study of the Chorin-Marsden formula, Math. Comp. 72 (2003), 307-333.

27. E. ZuAZUA, Log-Lipschitz regularity and uniqueness of the flow for a field in $\left(W_{\text {loc }}^{n / p+1, p}\left(\mathbb{R}^{n}\right)\right)^{n}$, C. R. Acad. Sci. Paris Sér. I 335 (2002), 17-22. 